\title{
ASYMPTOTIC ANALYSIS OF OPTIMIZED SCHWARZ METHODS FOR MAXWELL'S EQUATIONS WITH DISCONTINUOUS COEFFICIENTS
}

\author{
Victorita Dolean ${ }^{1}$, Martin J. Gander ${ }^{2}$ and ERwin Veneros ${ }^{3}$
}

\begin{abstract}
Discretized time harmonic Maxwell's equations are hard to solve by iterative methods, and the best currently available methods are based on domain decomposition and optimized transmission conditions. Optimized Schwarz methods were the first ones to use such transmission conditions, and this approach turned out to be so fundamentally important that it has been rediscovered over the last years under the name sweeping preconditioners, source transfer, single layer potential method and the method of polarized traces. We show here how one can optimize transmission conditions to take benefit from the jumps in the coefficients of the problem, when they are aligned with the subdomain interface, and obtain methods which converge for two subdomains in certain situations independently of the mesh size, which would not be possible without jumps in the coefficients.
\end{abstract}

1991 Mathematics Subject Classification. 65N55,65F10.

The dates will be set by the publisher.

\section{INTRODUCTION}

Time harmonic wave propagation problems are well known to be challenging to solve by iterative methods, for an overview in the case of the Helmholtz equation, see [12]. The most promising iterative algorithms are based on domain decomposition methods. After the seminal work in the PhD thesis [5] of Deprés, who devised an algorithm with Robin transmission conditions and proved its convergence, substantial progress has been made, leading to the class of optimized Schwarz methods, see $[3,4,13,14,16]$, and the more recent algorithms of sweeping type, source transfer, single layer potential and polarized traces use the same underlying ideas of optimized Schwarz methods, see [15] and references therein. Time harmonic Maxwell's equations inherit all these difficulties from the Helmholtz equation, and the unknown becomes in addition vector valued. Nevertheless, optimized Schwarz methods have been successfully also developed for Maxwell's equations, see $[1,8,18,19]$ for the case without conductivity and $[6,10]$ for the case with conductivity, where one sees that the presence of a nonzero conductivity is beneficial for convergence. For discretizations of Maxwell's equations using Discontinuous Galerkin methods, results on optimized Schwarz solvers can be found in [7,9], and for scattering problems and large scale applications, see $[18,19]$.

We are interested here in Maxwell's equations in the presence of jumps in the coefficients. We study in particular the case where the jumps can be aligned with subdomain interfaces, and show that then jumps can actually help convergence of the optimized Schwarz method, if the transmission conditions are appropriately

Keywords and phrases: Domain Decomposition, Maxwell's Equations, Discontinuous Coefficients, Optimized Schwarz Methods

${ }^{1}$ University of Strathclyde, Dept. of Maths and Stats, Glasgow, UK and Université Côte d'Azur, CNRS, LJAD, France

2 Section of Mathematics, University of Geneva, Switzerland

3 Section of Mathematics, University of Geneva, Switzerland 
chosen. In the presence of coefficients jumps, it is natural to consider non-overlapping Schwarz methods, and we study here the particular case of the 2d Maxwell's equations for TMz and TEz modes. For a special case in $2 \mathrm{~d}$, a convergence result stated without proof in [20] showed that well chosen transmission conditions can lead to non-overlapping Schwarz algorithms that converge independently of the mesh parameter. In addition it was shown in [21] that complete results for the $2 \mathrm{~d}$ transverse magnetic $(\mathrm{TMz})$ and transverse electric (TEz) modes ${ }^{1}$ will imply directly convergence results in $3 \mathrm{~d}$ as well. The purpose of this manuscript is to prove the $2 \mathrm{~d}$ results announced in [20] using asymptotic analysis. The analysis is very technical, and many cases need to be considered. We therefore give all the details only for the first case, and then only outline the most important technical steps for the remaining cases. We finally illustrate our results with numerical experiments.

\section{Schwarz Methods for Maxwell's Equations}

We consider in this paper the time-harmonic Maxwell equations with appropriate boundary conditions

$$
-i \omega \varepsilon \mathbf{E}+\nabla \times \mathbf{H}=\mathbf{J}, \quad i \omega \mu \mathbf{H}+\nabla \times \mathbf{E}=\mathbf{0}, \quad \text { in } \Omega,
$$

and we study the heterogeneous case where the domain $\Omega$ consists of two non-overlapping sub-domains $\Omega_{1}$ and $\Omega_{2}$ with interface $\Gamma:=\bar{\Omega}_{1} \cap \bar{\Omega}_{2}$, with piecewise constant electric permittivity $\varepsilon_{j}$ and piecewise constant magnetic permeability $\mu_{j}$ in $\Omega_{j}, j=1,2$. A general parallel Schwarz algorithm for these two non-overlapping subdomains $\Omega_{1}$ and $\Omega_{2}$ would start with some initial guess $\mathbf{E}^{j, 0}$ and $\mathbf{H}^{j, 0}$ on subdomain $\Omega_{j}$ and then compute for iteration index $n=1,2$,

$$
\left\{\begin{aligned}
-i \omega \varepsilon_{1} \mathbf{E}^{1, n}+\nabla \times \mathbf{H}^{1, n} & =\mathbf{J} & & \text { in } \Omega_{1}, \\
i \omega \mu_{1} \mathbf{H}^{1, n}+\nabla \times \mathbf{E}^{1, n} & =\mathbf{0} & & \text { in } \Omega_{1}, \\
\left(\mathcal{B}_{\mathbf{n}_{1}}+\mathcal{S}_{1} \mathcal{B}_{\mathbf{n}_{2}}\right)\left(\mathbf{E}^{1, n}, \mathbf{H}^{1, n}\right) & =\left(\mathcal{B}_{\mathbf{n}_{1}}+\mathcal{S}_{1} \mathcal{B}_{\mathbf{n}_{2}}\right)\left(\mathbf{E}^{2, n-1}, \mathbf{H}^{2, n-1}\right) & & \text { on } \Gamma \\
-i \omega \varepsilon_{2} \mathbf{E}^{2, n}+\nabla \times \mathbf{H}^{2, n} & =\mathbf{J} & & \text { in } \Omega_{2}, \\
i \omega \mu_{2} \mathbf{H}^{2, n}+\nabla \times \mathbf{E}^{2, n} & =\mathbf{0} & & \text { in } \Omega_{2}, \\
\left(\mathcal{B}_{\mathbf{n}_{2}}+\mathcal{S}_{2} \mathcal{B}_{\mathbf{n}_{1}}\right)\left(\mathbf{E}^{2, n}, \mathbf{H}^{2, n}\right) & =\left(\mathcal{B}_{\mathbf{n}_{2}}+\mathcal{S}_{2} \mathcal{B}_{\mathbf{n}_{1}}\right)\left(\mathbf{E}^{1, n-1}, \mathbf{H}^{1, n-1}\right) & & \text { on } \Gamma,
\end{aligned}\right.
$$

where $\mathcal{S}_{j}, j=1,2$ are tangential operators which will be differential or pseudo-differential, and

$$
\mathcal{B}_{\mathbf{n}_{j}}\left(\mathbf{E}^{j, n}, \mathbf{H}^{j, n}\right)=\frac{\mathbf{E}^{j, n}}{Z_{j}} \times \mathbf{n}_{j}+\mathbf{n}_{j} \times\left(\mathbf{H}^{j, n} \times \mathbf{n}_{j}\right), \quad Z_{j}:=\sqrt{\mu_{j} / \varepsilon_{j}},
$$

are the impedance conditions, and $\mathbf{n}_{j}$ is the unit outward normal for domain $\Omega_{j}, j=1,2$. Different choices of $\mathcal{S}_{j}, j=1,2$ lead to different Schwarz methods. Since it was shown in [21] that it is sufficient to study the $2 \mathrm{~d}$ $\mathrm{TMz}$ and $\mathrm{TEz}$ variants of (2) to get a complete understanding of (2), we focus in what follows on the $2 \mathrm{~d}$ case.

\subsection{Schwarz methods for the TMz and TEz modes}

We now present algorithm (2) for the TMz and TEz modes in two spatial dimensions. Since our analysis will be performed on the error equations, we directly state the algorithms for the homogeneous problems. For the $\mathrm{TMz}$ case, we obtain from (2)

$$
\left\{\begin{aligned}
i \omega \varepsilon_{1} E_{z}^{1, n}-\partial_{x} H_{y}^{1, n}+\partial_{y} H_{x}^{1, n} & =0 & & \text { in } \Omega_{1}, \\
i \omega \mu_{1} H_{x}^{1, n}+\partial_{y} E_{z}^{1, n} & =0 & & \text { in } \Omega_{1}, \\
i \omega \mu_{1} H_{y}^{1, n}-\partial_{x} E_{z}^{1, n} & =0 & & \text { in } \Omega_{1}, \\
\left(\mathcal{B}_{\mathbf{n}_{1}}+\mathcal{S}_{1} \mathcal{B}_{\mathbf{n}_{2}}\right)\left(E_{z}^{1, n}, H_{x}^{1, n}, H_{y}^{1, n}\right) & =\left(\mathcal{B}_{\mathbf{n}_{1}}+\mathcal{S}_{1} \mathcal{B}_{\mathbf{n}_{2}}\right)\left(E_{z}^{2, n-1}, H_{x}^{2, n-1}, H_{y}^{2, n-1}\right) & & \text { on } \Gamma, \\
i \omega \varepsilon_{2} E_{z}^{2, n}-\partial_{x} H_{y}^{2, n}+\partial_{y} H_{x}^{2, n} & =0 & & \text { in } \Omega_{2}, \\
i \omega \mu_{2} H_{x}^{2, n}+\partial_{y} E_{z}^{2, n} & =0 & & \text { in } \Omega_{2}, \\
i \omega \mu_{2} H_{y}^{2, n}-\partial_{x} E_{z}^{2, n} & =0 & & \text { in } \Omega_{2}, \\
\left(\mathcal{B}_{\mathbf{n}_{2}}+\mathcal{S}_{2} \mathcal{B}_{\mathbf{n}_{1}}\right)\left(E_{z}^{2, n}, H_{x}^{2, n}, H_{y}^{2, n}\right) & =\left(\mathcal{B}_{\mathbf{n}_{2}}+\mathcal{S}_{2} \mathcal{B}_{\mathbf{n}_{1}}\right)\left(E_{z}^{1, n-1}, H_{x}^{1, n-1}, H_{y}^{1, n-1}\right) & & \text { on } \Gamma .
\end{aligned}\right.
$$

\footnotetext{
${ }^{1}$ For a detailed introduction to the TMz and TEz equations see [17]
} 
which was obtained from (3) by replacing $\mathbf{E}^{j, n}=\left(0,0, E_{z}^{j, n}\right)$ and $\mathbf{H}^{j, n}=\left(H_{x}^{j, n}, H_{y}^{j, n}, 0\right)$. For the TEz case, we get

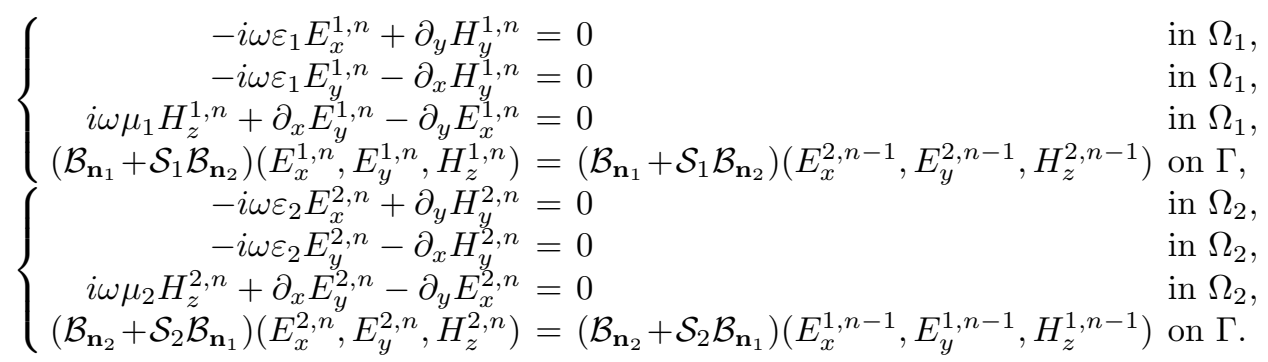

which we obtained from (3) by replacing $\mathbf{E}^{j, n}=\left(E_{x}^{j, n}, E_{y}^{j, n}, 0\right)$ and $\mathbf{H}^{j, n}=\left(0,0, H_{z}^{j, n}\right)$.

Remark 1. From the TEz equations we see that the TEz mode is related to the TMz mode, we just have to exchange the variables $H_{z}$ with $-E_{z}, E_{x}$ with $H_{x}$ and $E_{y}$ with $H_{y}$, and we get the same algorithm, provided we also switch $\mu$ and $\varepsilon$. It therefore suffices to either analyze the TMz or the TEz case, the results for the other case then follow by switching the roles of $\mu$ and $\varepsilon$.

For the TMz case, we can obtain an optimal Schwarz algorithm that converges in two iterations by choosing $\mathcal{S}_{1}$ and $\mathcal{S}_{2}$ such that in the transmission conditions in (4), namely

$$
\begin{aligned}
\left(\mathcal{B}_{\mathbf{n}_{1}}+\mathcal{S}_{1} \mathcal{B}_{\mathbf{n}_{2}}\right)\left(E_{z}^{1, n+1}, \mathbf{H}^{1, n+1}\right) & =\left(\mathcal{B}_{\mathbf{n}_{1}}+\mathcal{S}_{1} \mathcal{B}_{\mathbf{n}_{2}}\right)\left(E_{z}^{2, n}, \mathbf{H}^{2, n}\right), \\
\left(\mathcal{B}_{\mathbf{n}_{2}}+\mathcal{S}_{2} \mathcal{B}_{\mathbf{n}_{1}}\right)\left(E_{z}^{2, n+1}, \mathbf{H}^{2, n+1}\right) & =\left(\mathcal{B}_{\mathbf{n}_{2}}+\mathcal{S}_{2} \mathcal{B}_{\mathbf{n}_{1}}\right)\left(E_{z}^{1, n}, \mathbf{H}^{1, n}\right),
\end{aligned}
$$

the right hand side becomes homogeneous after the first iteration, which implies after a Fourier transform in the $y$ direction with Fourier parameter $k$ that

$$
\widehat{\mathcal{S}}_{1}=-\frac{\lambda_{2}-i \omega_{2} Z^{-1}}{\lambda_{2}+i \omega_{2}}, \quad \widehat{\mathcal{S}}_{2}=-\frac{\lambda_{1}-i \omega_{1} Z}{\lambda_{1}+i \omega_{1}}
$$

with $\lambda_{j}:=\sqrt{k^{2}-\omega_{j}^{2}}$ and $\omega_{j}:=\omega \sqrt{\varepsilon_{j} \mu_{j}}$, see [8, Theorem 3.2] for more details in the constant coefficient case. The choice (7) corresponds to the so called transparent conditions that were pioneered by Engquist and Majda [11]. They are well defined for any value of the Fourier parameter $k$, but are computationally expensive to use because Fourier transforms have to be performed due to the square root terms.

\subsection{Optimized convergence factor and min-max problems}

As for absorbing boundary conditions [11], we propose to approximate the transparent conditions in (7) by

$$
\widehat{\mathcal{S}}_{1} \approx-\frac{s_{2}-i \omega_{2} Z^{-1}}{s_{2}+i \omega_{2}}, \quad \widehat{\mathcal{S}}_{2} \approx-\frac{s_{1}-i \omega_{1} Z}{s_{1}+i \omega_{1}}
$$

with $s_{1}$ and $s_{2}$ two complex parameters. Using the approximations of $\widehat{\mathcal{S}}_{1}$ and $\widehat{\mathcal{S}}_{2}$ from $(8)$ in the transmission conditions (6), we obtain after a by now standard computation, see [20] for details, the convergence factor

$$
\rho_{\mathrm{opt}}\left(k, \omega_{1}, \omega_{2}, \mu_{1}, \mu_{2}, s_{1}, s_{2}\right)=\left(\frac{\left(\lambda_{1}-s_{1}\right)\left(\lambda_{2}-s_{2}\right)}{\left(\lambda_{1}+s_{2} \mu_{1} / \mu_{2}\right)\left(\lambda_{2}+s_{1} \mu_{2} / \mu_{1}\right)}\right)^{\frac{1}{2}} .
$$

To get a fast algorithm, we have to chose the complex parameters $s_{1}$ and $s_{2}$ such that the convergence factor is minimized for all relevant numerical frequencies, i.e. we have to minimize $\left|\rho_{\text {opt }}\right|$ for all $k \in K:=\left[k_{\min }, k_{\max }\right]$, where $k_{\min }$ is the smallest relevant frequency $\left(k_{\min }\right.$ depends on the geometry of the domain) and $k_{\max }$ is the 
largest frequency supported by the numerical grid (if the grid size $h$ is constant we have $k_{\max }=\frac{c_{\max }}{h}$, where $c_{\max }$ is some constant, often estimated by $\pi$ ). This leads to the min-max problem

$$
\min _{s_{1}, s_{2} \in \mathbb{C}} \max _{k \in K}\left|\rho_{\text {opt }}\left(k, \omega_{1}, \omega_{2}, \mu_{1}, \mu_{2}, s_{1}, s_{2}\right)\right| \text {. }
$$

Remark 2. For the TEz case we obtain the convergence factor

$$
\rho_{\text {opt }}\left(k, \omega_{1}, \omega_{2}, \varepsilon_{1}, \varepsilon_{2}, s_{1}, s_{2}\right)=\left(\frac{\left(\lambda_{1}-s_{1}\right)\left(\lambda_{2}-s_{2}\right)}{\left(\lambda_{1}+s_{2} \varepsilon_{1} / \varepsilon_{2}\right)\left(\lambda_{2}+s_{1} \varepsilon_{2} / \varepsilon_{1}\right)}\right)^{\frac{1}{2}}
$$

and the equivalent min-max problem

$$
\min _{s_{1}, s_{2} \in \mathbb{C}} \max _{k \in K}\left|\rho_{\text {opt }}\left(k, \omega_{1}, \omega_{2}, \varepsilon_{1}, \varepsilon_{2}, s_{1}, s_{2}\right)\right|
$$

We assume in what follows that the parameters are of the form $s_{1}=(1+i) C_{1}, s_{2}=(1+i) C_{2}$, a choice that was justified for Helmholtz equations in [16] and Maxwell's equations in [2].

To study the min-max problem (10), we have to divide the frequency interval $K$ into three sub intervals $\left(\left[0, \omega_{\min }\right],\left[\omega_{\min }, \omega_{\max }\right],\left[\omega_{\max }, k_{\max }\right]\right)$, where $\omega_{\min }:=\min \left\{\omega_{1}, \omega_{2}\right\}$ and $\omega_{\max }:=\max \left\{\omega_{1}, \omega_{2}\right\}$. These three inter-

vals are implied by the change of $\lambda_{1}$ and $\lambda_{2}$ from imaginary to real. We only consider the case $\omega_{1} \leq \omega_{2}$ because the other results follow by symmetry of $(9)$. For $k \in\left[0, \omega_{1}\right]$, the convergence factor in (9) is equal to

$$
\rho_{\mathrm{opt}}\left(k, \omega_{1}, \omega_{2}, \mu_{1}, \mu_{2}, C_{1}, C_{2}\right)=\frac{\left(i \tilde{\lambda}_{1}-(1+i) C_{1}\right)\left(i \tilde{\lambda}_{2}-(1+i) C_{2}\right)}{\left(i \tilde{\lambda}_{1}+\frac{\mu_{1}}{\mu_{2}}(1+i) C_{2}\right)\left(i \tilde{\lambda}_{2}+\frac{\mu_{2}}{\mu_{1}}(1+i) C_{1}\right)},
$$

with $\tilde{\lambda}_{j}:=\sqrt{\omega_{j}^{2}-k^{2}}$. For $k \in\left[\omega_{1}, \omega_{2}\right]$ we have

$$
\rho_{\mathrm{opt}}\left(k, \omega_{1}, \omega_{2}, \mu_{1}, \mu_{2}, C_{1}, C_{2}\right)=\frac{\left(\lambda_{1}-(1+i) C_{1}\right)\left(i \tilde{\lambda}_{2}-(1+i) C_{2}\right)}{\left(\lambda_{1}+\frac{\mu_{1}}{\mu_{2}}(1+i) C_{2}\right)\left(i \tilde{\lambda}_{2}+\frac{\mu_{2}}{\mu_{1}}(1+i) C_{1}\right)} .
$$

Finally, for $k \in\left[\omega_{2}, k_{\max }\right]$ we have

$$
\rho_{\mathrm{opt}}\left(k, \omega_{1}, \omega_{2}, \mu_{1}, \mu_{2}, C_{1}, C_{2}\right)=\frac{\left(\lambda_{1}-(1+i) C_{1}\right)\left(\lambda_{2}-(1+i) C_{2}\right)}{\left(\lambda_{1}+\frac{\mu_{1}}{\mu_{2}}(1+i) C_{2}\right)\left(\lambda_{2}+\frac{\mu_{2}}{\mu_{1}}(1+i) C_{1}\right)} .
$$

In nature the magnetic permeability $\mu$ is almost constant and the rate of change of the magnetic permeability $\mu$ for different materials can be neglected in comparison to the rate of change of the electric permittivity $\varepsilon$. We thus present here the case $\mu_{1}=\mu_{2}$ and $\varepsilon_{1} \neq \varepsilon_{2}$ both for the TMz and the TEz mode. Using Remark 1, one can then also read off the corresponding results for the physically less important case $\mu_{1} \neq \mu_{2}$ and $\varepsilon_{1}=\varepsilon_{2}$. The case where both coefficients have jumps can also be treated under an additional hypothesis, see [22, Theorem 2.5.1].

\section{TRANSMission CONDITIONS FOR THE TMz MODE}

The condition $\varepsilon_{1} \neq \varepsilon_{2}$ implies that $\omega_{1} \neq \omega_{2}$. The case $\omega_{1}=\omega_{2}$ is a resonance case that was studied in [22] and needs special treatment, like the particular resonance case where $\varepsilon$ and $\mu$ are continuous, see [8]. The fact to have a jump in $\varepsilon$ helps convergence, and Theorem 3 below was presented in a less general form in [20] without proof, and was the main building block to understand the $3 \mathrm{~d}$ case in [21]. We give now the general result, and a detailed proof based on asymptotic analysis. 
Theorem 3. If $\mu_{1}=\mu_{2}, \varepsilon_{1} \neq \varepsilon_{2}, s_{1}=(1+i) C_{1}, s_{2}=(1+i) C_{2}$ and $r=\sqrt{\left|\omega_{1}^{2}-\omega_{2}^{2}\right|}$, then the solution of the min-max problem (10) for $h$ small is given by

$$
\begin{gathered}
C_{1}^{*}=\left(\frac{r}{2}\right)^{\frac{1}{4}}\left(\frac{c_{\max }}{h}\right)^{\frac{3}{4}}, \quad C_{2}^{*}=\frac{1}{2}\left(\frac{r}{2}\right)^{\frac{3}{4}}\left(\frac{c_{\max }}{h}\right)^{\frac{1}{4}}, \\
\rho_{o p t}^{*}=1-\left(\frac{r}{2 c_{\max }}\right)^{\frac{1}{4}} h^{\frac{1}{4}}+\mathcal{O}\left(h^{\frac{1}{2}}\right),
\end{gathered}
$$

and the roles of $s_{1}$ and $s_{2}$ can also be reversed.

Proof. We set $C_{1}:=\frac{c_{1}}{h^{\alpha}}$ and $C_{2}:=\frac{c_{2}}{h^{\beta}}$, and determine the exponents and constants which solve the min-max problem (10). We divide the proof into three cases (Case I: $\beta<\alpha$, Case II: $\beta=\alpha$, and Case III: $\beta>\alpha$ ). In every case we will perform the following steps:

(1) Search and classify the extrema for $k=c$ constant.

(2) Verify that we do not have an extremum for $k$ close to 0 , which means for $k=c_{m} h^{\gamma}$, with $\gamma>0$.

(3) Search and classify the variable extrema for $k=\frac{c_{m}}{h \gamma}$ with $0 \leq \gamma \leq 1$.

(4) Compare the possible maxima for the values found in the previous steps and balance them to solve the min-max problem (10).

Remark 4. The constant $\gamma$ can not be greater than 1 because $k_{\max }=\frac{c_{\max }}{h}$ and we are not interested in higher frequencies than $k_{\max }$.

Case I $(\beta<\alpha)$ :

1. To show asymptotically that we only have one local extremum for $k$ constant, we have to study the convergence factor in the three intervals $(13,14,15)$ : if $k \in\left[0, \omega_{1}\right]$ we obtain for the modulus squared of the convergence factor in (13) as a function of $C_{1}$ and $C_{2}$

$$
R_{1}\left(k, \omega_{1}, \omega_{2}, C_{1}, C_{2}\right):=\frac{\left(\frac{c_{1}^{2}}{h^{2 \alpha}}+\left(\tilde{\lambda}_{1}-\frac{c_{1}}{h^{\alpha}}\right)^{2}\right)\left(\frac{c_{2}^{2}}{h^{2 \beta}}+\left(\tilde{\lambda}_{2}-\frac{c_{2}}{h^{\beta}}\right)^{2}\right)}{\left(\frac{c_{2}^{2}}{h^{2 \beta}}+\left(\tilde{\lambda}_{1}+\frac{c_{2}}{h^{\beta}}\right)^{2}\right)\left(\frac{c_{1}^{2}}{h^{2 \alpha}}+\left(\tilde{\lambda}_{2}+\frac{c_{1}}{h^{\alpha}}\right)^{2}\right)}
$$

Taking a partial derivative with respect to $k$, we get

$$
\frac{d R_{1}}{d k}\left(k, \omega_{1}, \omega_{2}, C_{1}, C_{2}\right)=R_{11}+R_{12}+R_{13}+R_{14}
$$

where

$$
\begin{aligned}
& R_{11}=\frac{-\frac{2 k}{\tilde{\lambda}_{1}}\left(\tilde{\lambda}_{1}-\frac{c_{1}}{h^{\alpha}}\right)\left(\frac{c_{2}^{2}}{h^{2 \beta}}+\left(\tilde{\lambda}_{2}-\frac{c_{2}}{h^{\beta}}\right)^{2}\right)}{\left(\frac{c_{2}^{2}}{h^{2 \beta}}+\left(\tilde{\lambda}_{1}+\frac{c_{2}}{h^{\beta}}\right)^{2}\right)\left(\frac{c_{1}^{2}}{h^{2 \alpha}}+\left(\tilde{\lambda}_{2}+\frac{c_{1}}{h^{\alpha}}\right)^{2}\right)}, \\
& R_{12}=\frac{-\frac{2 k}{\tilde{\lambda}_{2}}\left(\tilde{\lambda}_{2}-\frac{c_{2}}{h^{\beta}}\right)\left(\frac{c_{1}^{2}}{h^{2 \alpha}}+\left(\tilde{\lambda}_{1}-\frac{c_{1}}{h^{\alpha}}\right)^{2}\right)}{\left(\frac{c_{2}^{2}}{h^{2 \beta}}+\left(\tilde{\lambda}_{1}+\frac{c_{2}}{h^{\beta}}\right)^{2}\right)\left(\frac{c_{1}^{2}}{h^{2 \alpha}}+\left(\tilde{\lambda}_{2}+\frac{c_{1}}{h_{2}^{\alpha}}\right)^{2}\right)}, \\
& R_{13}=\frac{\frac{2 k}{\tilde{\lambda}_{1}}\left(\tilde{\lambda}_{1}+\frac{c_{2}}{h^{\beta}}\right)\left(\frac{c_{1}^{2}}{h^{2 \alpha}}+\left(\tilde{\lambda}_{1}-\frac{c_{1}}{h^{\alpha}}\right)^{2}\right)\left(\frac{c_{2}^{2}}{h^{2 \beta}}+\left(\tilde{\lambda}_{2}-\frac{c_{2}}{h^{\beta}}\right)^{2}\right)}{\left(\frac{c_{2}^{2}}{h^{2 \beta}}+\left(\tilde{\lambda}_{1}+\frac{c_{2}}{h^{\beta}}\right)^{2}\right)^{2}\left(\frac{c_{1}^{2}}{h^{2 \alpha}}+\left(\tilde{\lambda}_{2}+\frac{c_{1}}{h^{\alpha}}\right)^{2}\right)}, \\
& R_{14}=\frac{\frac{2 k}{\tilde{\lambda}_{2}}\left(\tilde{\lambda}_{2}+\frac{c_{1}}{h^{\alpha}}\right)\left(\frac{c_{1}^{2}}{h^{2 \alpha}}+\left(\tilde{\lambda}_{1}-\frac{c_{1}}{h^{\alpha}}\right)^{2}\right)\left(\frac{c_{2}^{2}}{h^{2 \beta}}+\left(\tilde{\lambda}_{2}-\frac{c_{2}}{h^{\beta}}\right)^{2}\right)}{\left(\frac{c_{2}^{2}}{h^{2 \beta}}+\left(\tilde{\lambda}_{1}+\frac{c_{2}}{h^{\beta}}\right)^{2}\right)\left(\frac{c_{1}^{2}}{h^{2 \alpha}}+\left(\tilde{\lambda}_{2}+\frac{c_{1}}{h^{\alpha}}\right)^{2}\right)^{2}} .
\end{aligned}
$$


In order to evaluate $R_{1}$ in (17) and $\frac{d R_{1}}{d k}$ in (19) asymptotically, we need to expand all terms in (19), which leads to

$$
\begin{aligned}
\left(\tilde{\lambda}_{1}-\frac{c_{1}}{h^{\alpha}}\right) & \simeq-\frac{c_{1}}{h^{\alpha}}\left(1-\frac{\tilde{\lambda}_{1}}{c_{1}} h^{\alpha}\right), \\
\left(\tilde{\lambda}_{2}-\frac{c_{2}}{h^{\beta}}\right) & \simeq-\frac{c_{2}}{h^{\beta}}\left(1-\frac{\tilde{\lambda}_{2}}{c_{2}} h^{\beta}\right), \\
\left(\tilde{\lambda}_{1}+\frac{c_{2}}{h^{\beta}}\right) & \simeq \frac{c_{2}}{h^{\beta}}\left(1+\frac{\tilde{\lambda}_{1}}{c_{2}} h^{\beta}\right), \\
\left(\tilde{\lambda}_{2}+\frac{c_{1}}{h^{\alpha}}\right) & \simeq \frac{c_{1}}{h^{\alpha}}\left(1+\frac{\tilde{\lambda}_{2}}{c_{1}} h^{\alpha}\right), \\
\left(\frac{c_{1}^{2}}{h^{2 \alpha}}+\left(\tilde{\lambda}_{1}-\frac{c_{1}}{h^{\alpha}}\right)^{2}\right) & \simeq \frac{2 c_{1}^{2}}{h^{2 \alpha}}\left(1-\frac{\tilde{\lambda}_{1}}{c_{1}} h^{\alpha}+\mathcal{O}\left(h^{2 \alpha}\right)\right), \\
\left(\frac{c_{2}^{2}}{h^{2 \beta}}+\left(\tilde{\lambda}_{2}-\frac{c_{2}}{h^{\beta}}\right)^{2}\right) & \simeq \frac{2 c_{2}^{2}}{h^{2 \beta}}\left(1-\frac{\tilde{\lambda}_{2}}{c_{2}} h^{\beta}+\mathcal{O}\left(h^{2 \beta}\right)\right), \\
\left(\frac{c_{2}^{2}}{h^{2 \beta}}+\left(\tilde{\lambda}_{1}+\frac{c_{2}}{h^{\beta}}\right)^{2}\right) & \simeq \frac{2 c_{2}^{2}}{h^{2 \beta}}\left(1+\frac{\tilde{\lambda}_{1}}{c_{2}} h^{\beta}+\mathcal{O}\left(h^{2 \beta}\right)\right), \\
\left(\frac{c_{1}^{2}}{h^{2 \alpha}}+\left(\tilde{\lambda}_{2}+\frac{c_{1}}{h^{\alpha}}\right)^{2}\right) & \simeq \frac{c_{1}^{2}}{h^{2 \alpha}}\left(1+\frac{\tilde{\lambda}_{2}}{c_{1}} h^{\alpha}+\mathcal{O}\left(h^{2 \alpha}\right)\right), \\
\left(\frac{c_{2}^{2}}{h^{2 \beta}}+\left(\tilde{\lambda}_{1}+\frac{c_{2}}{h^{\beta}}\right)^{2}\right)^{-1} & \simeq \frac{h^{2 \beta}}{2 c_{2}^{2}}\left(1-\frac{\tilde{\lambda}_{1}}{c_{2}} h^{\beta}+\mathcal{O}\left(h^{2 \beta}\right)\right), \\
\left(\frac{c_{1}^{2}}{h^{2 \alpha}}+\left(\tilde{\lambda}_{2}+\frac{c_{1}}{h^{\alpha}}\right)^{2}\right)^{-1} & \simeq \frac{h^{2 \alpha}}{2 c_{1}^{2}}\left(1-\frac{\tilde{\lambda}_{2}}{c_{1}} h^{\alpha}+\mathcal{O}\left(h^{2 \alpha}\right)\right), \\
\left(\frac{c_{2}^{2}}{h^{2 \beta}}+\left(\tilde{\lambda}_{1}+\frac{c_{2}}{h^{\beta}}\right)^{2}\right)^{-2} & \simeq \frac{h^{4 \beta}}{4 c_{2}^{4}}\left(1-\frac{2 \tilde{\lambda}_{1}}{c_{2}} h^{\beta}+\mathcal{O}\left(h^{2 \beta}\right)\right), \\
\left(\frac{c_{1}^{2}}{h^{2 \alpha}}+\left(\tilde{\lambda}_{2}+\frac{c_{1}}{h^{\alpha}}\right)^{2}\right)^{-2} & \simeq \frac{h^{4 \alpha}}{4 c_{1}^{4}}\left(1-\frac{2 \tilde{\lambda}_{2}}{c_{1}} h^{\alpha}+\mathcal{O}\left(h^{2 \alpha}\right)\right) .
\end{aligned}
$$

Replacing these expressions into (19) and collecting leading order terms, we get

$$
\begin{aligned}
& R_{11} \simeq\left(-\frac{2 k}{\tilde{\lambda}_{1}}\right)\left(-\frac{c_{1}}{h^{\alpha}}\right)\left(1-\frac{\tilde{\lambda}_{1}}{c_{1}} h^{\alpha}\right)\left(\frac{2 c_{2}^{2}}{h^{2 \beta}}\right)\left(1-\frac{\tilde{\lambda}_{2}}{c_{2}} h^{\beta}+\mathcal{O}\left(h^{2 \beta}\right)\right)\left(\frac{h^{2 \beta}}{2 c_{2}^{2}}\right) \\
& \left(1-\frac{\tilde{\lambda}_{1}}{c_{2}} h^{\beta}+\mathcal{O}\left(h^{2 \beta}\right)\right)\left(\frac{h^{2 \alpha}}{2 c_{1}^{2}}\right)\left(1-\frac{\tilde{\lambda}_{2}}{c_{1}} h^{\alpha}+\mathcal{O}\left(h^{2 \alpha}\right)\right) \\
& \simeq\left(\frac{k}{\tilde{\lambda}_{1} c_{1}} h^{\alpha}\right)\left(1-\frac{\tilde{\lambda}_{1}+\tilde{\lambda}_{2}}{c_{2}} h^{\beta}-\frac{\tilde{\lambda}_{1}+\tilde{\lambda}_{2}}{c_{1}} h^{\alpha}+\mathcal{O}\left(h^{2 \beta}\right)\right) \text {, } \\
& R_{12} \simeq\left(-\frac{2 k}{\tilde{\lambda}_{2}}\right)\left(-\frac{c_{2}}{h^{\beta}}\right)\left(1-\frac{\tilde{\lambda}_{2}}{c_{2}} h^{\beta}\right)\left(\frac{2 c_{1}^{2}}{h^{2 \alpha}}\right)\left(1-\frac{\tilde{\lambda}_{1}}{c_{1}} h^{\alpha}+\mathcal{O}\left(h^{2 \alpha}\right)\right)\left(\frac{h^{2 \beta}}{2 c_{2}^{2}}\right) \\
& \left(1-\frac{\tilde{\lambda}_{1}}{c_{2}} h^{\beta}+\mathcal{O}\left(h^{2 \beta}\right)\right)\left(\frac{h^{2 \alpha}}{2 c_{1}^{2}}\right)\left(1-\frac{\tilde{\lambda}_{2}}{c_{1}} h^{\alpha}+\mathcal{O}\left(h^{2 \alpha}\right)\right) \\
& \simeq\left(\frac{k}{\tilde{\lambda}_{2} c_{2}} h^{\beta}\right)\left(1-\frac{\tilde{\lambda}_{1}+\tilde{\lambda}_{2}}{c_{2}} h^{\beta}-\frac{\tilde{\lambda}_{1}+\tilde{\lambda}_{2}}{c_{1}} h^{\alpha}+\mathcal{O}\left(h^{2 \beta}\right)\right), \\
& R_{13} \simeq\left(\begin{array}{l}
2 k \\
\tilde{\lambda}_{1}
\end{array}\right)\left(\frac{c_{2}}{h^{\beta}}\right)\left(1+\frac{\tilde{\lambda}_{1}}{c_{2}} h^{\beta}\right)\left(\frac{2 c_{1}^{2}}{h^{2 \alpha}}\right)\left(1-\frac{\tilde{\lambda}_{1}}{c_{1}} h^{\alpha}+\mathcal{O}\left(h^{2 \alpha}\right)\right)\left(\frac{2 c_{2}^{2}}{h^{2 \beta}}\right) \\
& \left(\frac{h^{4 \beta}}{4 c_{2}^{4}}\right)\left(\frac{h^{2 \alpha}}{2 c_{1}^{2}}\right)\left(1-\frac{\tilde{\lambda}_{2}}{c_{2}} h^{\beta}+\mathcal{O}\left(h^{2 \beta}\right)\right)\left(1-\frac{2 \tilde{\lambda}_{1}}{c_{2}} h^{\beta}+\mathcal{O}\left(h^{2 \beta}\right)\right) \\
& \left(1-\frac{\tilde{\lambda}_{2}}{c_{1}} h^{\alpha}+\mathcal{O}\left(h^{2 \alpha}\right)\right) \\
& \simeq\left(\frac{k}{\tilde{\lambda}_{1} c_{2}} h^{\beta}\right)\left(1-\frac{\tilde{\lambda}_{1}+\tilde{\lambda}_{2}}{c_{2}} h^{\beta}-\frac{\tilde{\lambda}_{1}+\tilde{\lambda}_{2}}{c_{1}} h^{\alpha}+\mathcal{O}\left(h^{2 \beta}\right)\right), \\
& R_{14} \simeq\left(\frac{2 k}{\tilde{\lambda}_{2}}\right)\left(\frac{c_{1}}{h^{\alpha}}\right)\left(1+\frac{\tilde{\lambda}_{2}}{c_{1}} h^{\alpha}\right)\left(\frac{2 c_{1}^{2}}{h^{2 \alpha}}\right)\left(1-\frac{\tilde{\lambda}_{1}}{c_{1}} h^{\alpha}+\mathcal{O}\left(h^{2 \alpha}\right)\right)\left(\frac{2 c_{2}^{2}}{h^{2 \beta}}\right) \\
& \left(\frac{h^{2 \beta}}{2 c_{2}^{2}}\right)\left(\frac{h^{4 \alpha}}{4 c_{1}^{4}}\right)\left(1-\frac{\tilde{\lambda}_{2}}{c_{2}} h^{\beta}+\mathcal{O}\left(h^{2 \beta}\right)\right)\left(1-\frac{\tilde{\lambda}_{1}}{c_{2}} h^{\beta}+\mathcal{O}\left(h^{2 \beta}\right)\right) \\
& \left(1-\frac{2 \tilde{\lambda}_{2}}{c_{1}} h^{\alpha}+\mathcal{O}\left(h^{2 \alpha}\right)\right) \\
& \simeq\left(\frac{k}{\tilde{\lambda}_{2} c_{1}} h^{\alpha}\right)\left(1-\frac{\tilde{\lambda}_{1}+\tilde{\lambda}_{2}}{c_{2}} h^{\beta}-\frac{\tilde{\lambda}_{1}+\tilde{\lambda}_{2}}{c_{1}} h^{\alpha}+\mathcal{O}\left(h^{2 \beta}\right)\right) \text {. }
\end{aligned}
$$

We thus obtain for the asymptotic behavior of $\frac{d R_{1}}{d k}$

$$
\frac{d R_{1}}{d k}\left(k, \omega_{1}, \omega_{2}, C_{1}, C_{2}\right)=k\left(\frac{1}{\tilde{\lambda}_{1}}+\frac{1}{\tilde{\lambda}_{2}}\right)\left[\frac{1}{c_{2}} h^{\beta}+\frac{1}{c_{1}} h^{\alpha}+\mathcal{O}\left(h^{2 \beta}\right)\right]
$$


and hence there is a local extremum at $k=0$. For $k \in\left(0, \omega_{1}\right)$ we have $\frac{d R_{1}}{d k}>0$, because $\left(\frac{1}{\tilde{\lambda}_{1}}+\frac{1}{\tilde{\lambda}_{2}}\right)>0$ for all $k \in\left(0, \omega_{1}\right)$, so there are no other local extrema in $\left(0, \omega_{1}\right)$ for $k$ fixed and thus $k=0$ is a minimum and $k=\omega_{1}$ is a maximum.

Performing a similar study for the interval $\left[\omega_{1}, \omega_{2}\right]$, for details see [22], we obtain

$$
\frac{d R_{2}}{d k}\left(k, \omega_{1}, \omega_{2}, C_{1}, C_{2}\right)=k\left(\frac{\lambda_{1}-\tilde{\lambda}_{2}}{\lambda_{1} \tilde{\lambda}_{2}}\right)\left[\frac{1}{c_{2}} h^{\beta}+\frac{1}{c_{1}} h^{\alpha}+\mathcal{O}\left(h^{2 \beta}\right)\right] .
$$

Here the leading order term can vanish if $\tilde{\lambda}_{2}=\lambda_{1}$, i.e. when

$$
k=k_{1}:=\sqrt{\frac{\omega_{1}^{2}+\omega_{2}^{2}}{2}} .
$$

If $k<\sqrt{\frac{\omega_{1}^{2}+\omega_{2}^{2}}{2}}$ we have $\lambda_{1}<\tilde{\lambda}_{2}$ and $\frac{d R_{2}}{d k}<0$, and if $k>\sqrt{\frac{\omega_{1}^{2}+\omega_{2}^{2}}{2}}$ we have $\lambda_{1}>\tilde{\lambda}_{2}$ and thus $\frac{d R_{2}}{d k}>0$. Therefore $k_{1}$ is a local minimum, not a maximum. The maximum on the interval is thus either at $k=\omega_{1}$ or $k=\omega_{2}$.

Now we verify that we do not have extrema for $k$ fixed in the third interval $\left[\omega_{2}, k_{\text {max }}\right]$. With similar computations as before, see [22] for details, we obtain

$$
\frac{d R_{3}}{d k}\left(k, \omega_{1}, \omega_{2}, C_{1}, C_{2}\right)=k\left(\frac{\lambda_{1}+\lambda_{2}}{\lambda_{1} \lambda_{2}}\right)\left[-\frac{1}{c_{2}} h^{\beta}-\frac{1}{c_{1}} h^{\alpha}+\mathcal{O}\left(h^{2 \beta}\right)\right] .
$$

Since $\frac{\lambda_{1}+\lambda_{2}}{\lambda_{1} \lambda_{2}}>0$ for $k \in\left[\omega_{2}, k_{\max }\right]$, it follows that $R_{3}$ does not have an extremum for fixed $k \in\left[\omega_{2}, k_{\max }\right]$, and the sign of the derivative shows that $R_{3}$ is decreasing for $k \geq \omega_{2}$.

2. Now we show that there is no variable extremum close to $k=0$ : we suppose that $k=c_{m} h^{\gamma}$, with $\gamma>0$, and obtain with a similar approach as before (for details, see [22]), that

$$
\frac{d R_{1}}{d k}\left(k, \omega_{1}, \omega_{2}, C_{1}, C_{2}\right)=k\left(\frac{1}{\omega_{1}}+\frac{1}{\omega_{2}}\right)\left[\frac{1}{c_{2}} h^{\beta}+\frac{1}{c_{1}} h^{\alpha}+\mathcal{O}\left(h^{*}\right)\right],
$$

with $*$ denoting a number bigger than $\beta$. The leading order term can not vanish, and we thus can not have further extrema close to $k=0$.

3. We now study possible extrema for $k=\frac{c_{m}}{h \gamma}$, with $0<\gamma \leq 1$. There are five sub-cases to consider: $\gamma<\beta<\alpha, \beta<\gamma<\alpha, \beta<\alpha<\gamma$ and also the two particular cases $\beta=\gamma<\alpha$ and $\beta<\gamma=\alpha$. For every case we have to do a similar calculation as we did for the case $k$ constant in the interval $\left[0, \omega_{1}\right]$. For $\gamma<\beta<\alpha$ we get (for details see [22])

$$
\frac{d R_{3}}{d k}\left(k, \omega_{1}, \omega_{2}, C_{1}, C_{2}\right)=-\frac{2}{c_{1}} h^{\beta}-\frac{2}{c_{2}} h^{\alpha}+\mathcal{O}\left(h^{\min \{2 \beta-\gamma, \beta+2 \gamma\}}\right) .
$$

This shows that the leading order term of (25) can not vanish by an appropriate choice of $\gamma$ and $c_{m}$, since it does not depend on them, and thus there is no local extremum for $\gamma<\beta<\alpha$. For $\beta<\alpha<\gamma$, we get

$$
\frac{d R_{3}}{d k}\left(k, \omega_{1}, \omega_{2}, C_{1}, C_{2}\right)=\frac{4}{c_{m}^{2}} h^{\gamma}\left(c_{1} h^{\gamma-\alpha}+c_{2} h^{\gamma-\beta}+\mathcal{O}\left(h^{2 \gamma-2 \alpha}\right)\right),
$$

and again the leading order term can not vanish by an appropriate choice of $\gamma$ and $c_{m}$; we can not have a local extremum either. For the case $k=c_{m} / h^{\gamma}$ and $\beta<\gamma<\alpha$, we get

$$
\frac{d R_{3}}{d k}\left(k, \omega_{1}, \omega_{2}, C_{1}, C_{2}\right)=\frac{4 c_{2}}{c_{m}^{2}} h^{2 \gamma-\beta}-\frac{2}{c_{1}} h^{\alpha}+\mathcal{O}\left(h^{\min \{2 \alpha-\gamma, \alpha+\gamma-\beta, 3 \gamma-2 \beta\}}\right) .
$$



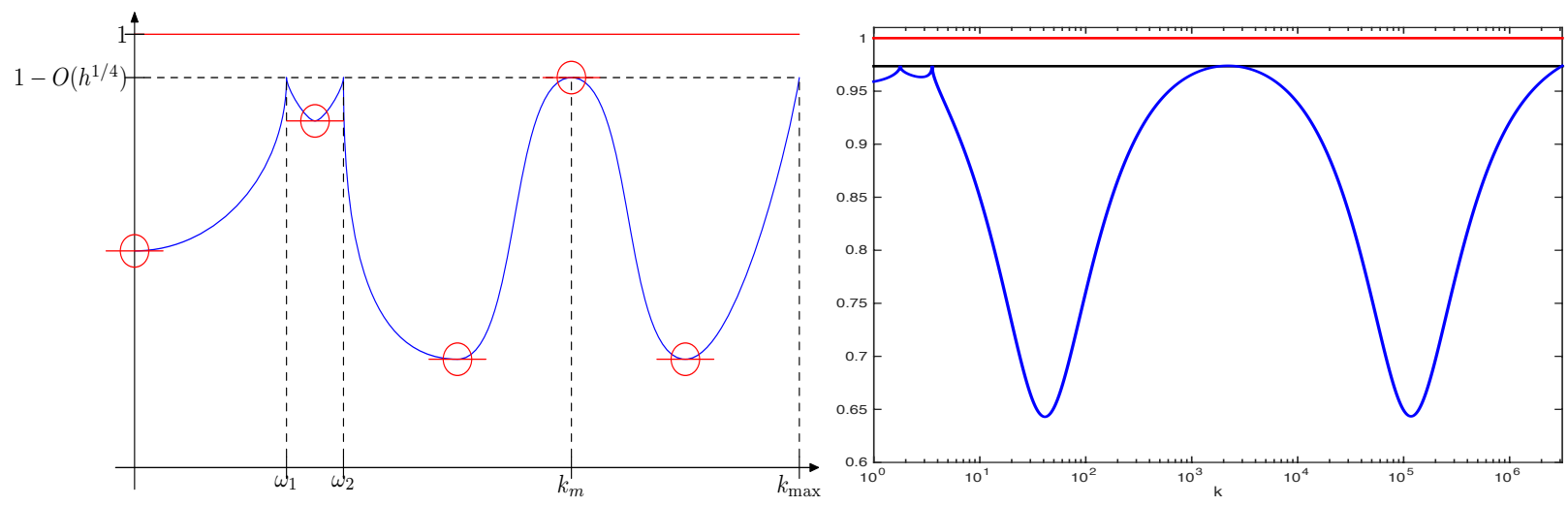

FigURE 1. Left: drawing of the optimized convergence factor $\rho_{\text {opt }}$ studied in Theorem 3. Right: actual plot of the optimized convergence factor from 3 for $\mu_{1}=\mu_{2}=1, \varepsilon_{1}=1, \varepsilon_{2}=4, \omega=\pi$ and $h=10^{-6}$. The red line is at 1 and the black line is the asymptotic maximum $\left(1-\mathcal{O}\left(h^{\frac{1}{4}}\right)\right.$ for this case).

Choosing $\gamma=(\alpha+\beta) / 2$, we obtain

$$
\frac{d R_{3}}{d k}\left(k, \omega_{1}, \omega_{2}, C_{1}, C_{2}\right)=\frac{4 c_{1} c_{2}-2 c_{m}^{2}}{c_{m}^{2} c_{1}} h^{\alpha}+\mathcal{O}\left(h^{(3 \alpha-\beta) / 2}\right),
$$

and hence the leading order term vanishes if we choose for the constant $c_{m}=\sqrt{2 c_{1} c_{2}}$. We thus have a local extremum, and it is a maximum after studying the signs of the expressions above. For $\beta=\gamma<\alpha$ we get

$$
\frac{d R_{3}}{d k}\left(k, \omega_{1}, \omega_{2}, C_{1}, C_{2}\right)=\left(\frac{2 c_{2}\left(c_{m}^{2}-2 c_{2}^{2}\right)}{\left(c_{2}^{2}+\left(c_{m}+c_{2}\right)^{2}\right)^{2}}\right) h^{\beta}+\mathcal{O}\left(h^{\min \{\alpha, 2 \alpha-\beta\}}\right),
$$

and the leading order term vanishes if $c_{m}=\sqrt{2} c_{2}$. We thus have a local extremum, but it is a local minimum after a study of the signs. Finally, for $k=\frac{c_{m}}{h^{\alpha}}$ in the case $\beta<\alpha=\gamma$, we obtain

$$
\frac{d R_{3}}{d k}\left(k, \omega_{1}, \omega_{2}, C_{1}, C_{2}\right)=\frac{4 c_{1}\left(c_{m}^{2}-2 c_{1}^{2}\right)}{\left(c_{1}^{2}+\left(c_{m}+c_{1}\right)^{2}\right)^{2}} h^{\alpha}+\mathcal{O}\left(h^{2 \alpha-\beta}\right),
$$

and the leading order term vanishes for $c_{m}=\sqrt{2} c_{1}$, which is however also a minimum after a study of the signs.

We therefore know now that asymptotically the possible maxima of the convergence factor are at $k=$ $\omega_{1}, \omega_{2}, k_{m}$ and $k_{\max }$, as illustrated in Figure 1 by a drawing and an actually computed example.

4. First we show that $R_{1}\left(\omega_{1}, \omega_{1}, \omega_{2}, C_{1}, C_{2}\right) \simeq R_{2}\left(\omega_{2}, \omega_{1}, \omega_{2}, C_{1}, C_{2}\right)$. To compute the asymptotic expansions, we use (17) and a similar expression for $R_{2}$, see [22] for details. For $k=\omega_{1}$ we have $\tilde{\lambda}_{1}=0$ and $\tilde{\lambda}_{2}=\sqrt{\omega_{2}^{2}-\omega_{1}^{2}}=$ : $r$. In order to simplify the notation we denote $R_{1}\left(k, \omega_{1}, \omega_{2}, C_{1}, C_{2}\right)$ by $R_{1}(k)$ unless we have to specify the other parameters. The asymptotic expansion of $R_{1}(k)$ for $k=\omega_{1}$ gives

$$
\begin{aligned}
R_{1}\left(\omega_{1}\right)= & \left(\frac{2 c_{1}^{2}}{h^{2 \alpha}}\right)\left(1+\mathcal{O}\left(h^{2 \alpha}\right)\right)\left(\frac{2 c_{2}^{2}}{h^{2 \beta}}\right)\left(1-\frac{r}{c_{2}} h^{\beta}+\mathcal{O}\left(h^{2 \beta}\right)\right) \\
& \times\left(\frac{h^{2 \beta}}{2 c_{2}^{2}}\right)\left(1+\mathcal{O}\left(h^{2 \beta}\right)\right)\left(\frac{h^{2 \alpha}}{2 c_{1}^{2}}\right)\left(1-\frac{r}{c_{1}} h^{\alpha}+\mathcal{O}\left(h^{2 \alpha}\right)\right) \\
= & 1-\frac{r}{c_{2}} h^{\beta}-\frac{r}{c_{1}} h^{\alpha}+\mathcal{O}\left(h^{2 \beta}\right) .
\end{aligned}
$$


For $k=\omega_{2}$ we have $\lambda_{1}=\sqrt{\omega_{2}^{2}-\omega_{1}^{2}}=r$ and $\tilde{\lambda}_{2}=0$, and we get

$$
\begin{aligned}
R_{2}\left(\omega_{2}\right)= & \left(\frac{2 c_{1}^{2}}{h^{2 \alpha}}\right)\left(1-\frac{r}{c_{1}} h^{\alpha}+\mathcal{O}\left(h^{2 \alpha}\right)\right)\left(\frac{2 c_{2}^{2}}{h^{2 \beta}}\right)\left(1+\mathcal{O}\left(h^{2 \beta}\right)\right) \\
& \times\left(\frac{h^{2 \beta}}{2 c_{2}^{2}}\right)\left(1-\frac{r}{c_{2}} h^{\beta}+\mathcal{O}\left(h^{2 \beta}\right)\right)\left(\frac{h^{2 \alpha}}{2 c_{1}^{2}}\right)\left(1+\mathcal{O}\left(h^{2 \alpha}\right)\right) \\
= & 1-\frac{r}{c_{2}} h^{\beta}-\frac{r}{c_{1}} h^{\alpha}+\mathcal{O}\left(h^{2 \beta}\right) .
\end{aligned}
$$

Hence (28) and (29) are asymptotically equal, and we only need to consider one of them to perform the optimization; we will use $R_{1}\left(\omega_{1}\right)$.

Now the possible maxima are $R_{1}\left(\omega_{1}, \omega_{1}, \omega_{2}, C_{1}, C_{2}\right), R_{3}\left(k_{m}, \omega_{1}, \omega_{2}, C_{1}, C_{2}\right)$ and $R_{3}\left(k_{\max }, \omega_{1}, \omega_{2}, C_{1}, C_{2}\right)$. For $R_{3}\left(k_{m}, \omega_{1}, \omega_{2}, C_{1}, C_{2}\right)$ we obtain with $k=k_{m}:=\frac{\sqrt{2 c_{1} c_{2}}}{h^{\alpha / 2+\beta / 2}}$ the asymptotic expansion

$$
\begin{aligned}
R_{3}\left(k_{m}\right)= & \left(\frac{c_{1}^{2}}{h^{2 \alpha}}\right)\left(1-\sqrt{\frac{2 c_{2}}{c_{1}}} h^{\frac{\alpha}{2}}-\frac{\beta}{2}+\mathcal{O}\left(h^{\alpha-\beta}\right)\right)\left(\frac{h^{\alpha+\beta}}{2 c_{1} c_{2}}\right)\left(1-\sqrt{\frac{2 c_{2}}{c_{1}}} h^{\frac{\alpha-\beta}{2}}+\mathcal{O}\left(h^{\alpha-\beta}\right)\right) \\
& \times\left(1-\sqrt{\frac{2 c_{2}}{c_{1}}} h^{\frac{\alpha-\beta}{2}}+\mathcal{O}\left(h^{\alpha-\beta}\right)\right)\left(\frac{h^{2 \alpha}}{c_{1}^{2}}\right)\left(\frac{2 c_{1} c_{2}}{h^{\alpha+\beta}}\right)\left(1-\sqrt{\frac{2 c_{2}}{c_{1}}} h^{\frac{\alpha-\beta}{2}}+\mathcal{O}\left(h^{\alpha-\beta}\right)\right) \\
= & 1-4 \sqrt{2} \sqrt{\frac{c_{2}}{c_{1}}} h^{\frac{\alpha}{2}-\frac{\beta}{2}}+\mathcal{O}\left(h^{\alpha-\beta}\right) .
\end{aligned}
$$

For $R_{3}\left(k_{\max }, \omega_{1}, \omega_{2}, C_{1}, C_{2}\right)$ we obtain with $k=\frac{c_{\max }}{h}$

$$
\begin{aligned}
R_{3}\left(k_{\max }\right)= & \left(\frac{c_{m}^{2}}{h^{2}}\right)\left(1-\frac{2 c_{1}}{c_{\max }} h^{1-\alpha}+\mathcal{O}\left(h^{2-2 \alpha}\right)\right)\left(\frac{c_{\max }^{2}}{h^{2}}\right)\left(1-\frac{2 c_{2}}{c_{\max }} h^{1-\beta}+\mathcal{O}\left(h^{2-2 \beta}\right)\right) \\
& \times\left(\frac{h^{2 \gamma}}{c_{\max }^{2}}\right)\left(1-\frac{2 c_{2}}{c_{\max }} h^{1-\beta}+\mathcal{O}\left(h^{2-2 \beta}\right)\right)\left(\frac{h^{2}}{c_{\max }^{2}}\right)\left(1-\frac{2 c_{1}}{c_{\max }} h^{1-\alpha}+\mathcal{O}\left(h^{2-2 \alpha}\right)\right. \\
= & 1-\frac{4 c_{1}}{c_{\max }} h^{1-\alpha}+\mathcal{O}\left(h^{\min \{1-\beta, 2-2 \alpha\}}\right) .
\end{aligned}
$$

We thus need to choose $\alpha$ and $\beta$ to minimize the maximum of (28), (30) and (31), i.e. the maximum of

$$
\begin{aligned}
R_{1}\left(\omega_{1}, \omega_{1}, \omega_{2}, C_{1}, C_{2}\right) & =1-\frac{r}{c_{2}} h^{\beta}+\mathcal{O}\left(h^{\min \{\alpha, 2 \beta\}}\right), \\
R_{3}\left(k_{m}, \omega_{1}, \omega_{2}, C_{1}, C_{2}\right) & =1-4 \sqrt{2} \sqrt{\frac{c_{2}}{c_{1}}} h^{\alpha / 2-\beta / 2}+\mathcal{O}\left(h^{\alpha-\beta}\right), \\
R_{3}\left(k_{\max }, \omega_{1}, \omega_{2}, C_{1}, C_{2}\right) & =1-\frac{4 c_{1}}{c_{\max }} h^{1-\alpha}+\mathcal{O}\left(h^{\min \{1-\beta, 2-2 \alpha\}}\right) .
\end{aligned}
$$

To make $R_{1}\left(\omega_{1}\right)$ small we need $\beta$ small, and to make $R_{3}\left(k_{m}\right)$ small we need $\beta$ large, which implies that equioscillation gives the minimum,

$$
\beta=\alpha / 2-\beta / 2 \Longleftrightarrow 3 \beta=\alpha
$$

Now to make $R_{3}\left(k_{\max }\right)$ small we need $\alpha$ large and to make $R_{3}\left(k_{m}\right)$ small we need $\alpha$ small, which implies again equioscillation for the minimum,

$$
1-\alpha=\alpha / 2-\beta / 2 \quad \Leftrightarrow \quad 3 \alpha-\beta=2 .
$$

The two equations thus imply $\alpha=3 / 4$ and $\beta=1 / 4$. We show an example of the three functions whose maximum we minimize in Figure 2, where the minimizing point is clearly visible. Using the same argument for equioscillation, we can also determine the constants, and find

$$
c_{1}^{*}=\left(\frac{r}{2}\right)^{\frac{1}{4}} c_{\max }^{\frac{3}{4}}, \quad c_{2}^{*}=\frac{1}{2}\left(\frac{r}{2}\right)^{\frac{3}{4}} c_{\max }^{\frac{1}{4}} .
$$




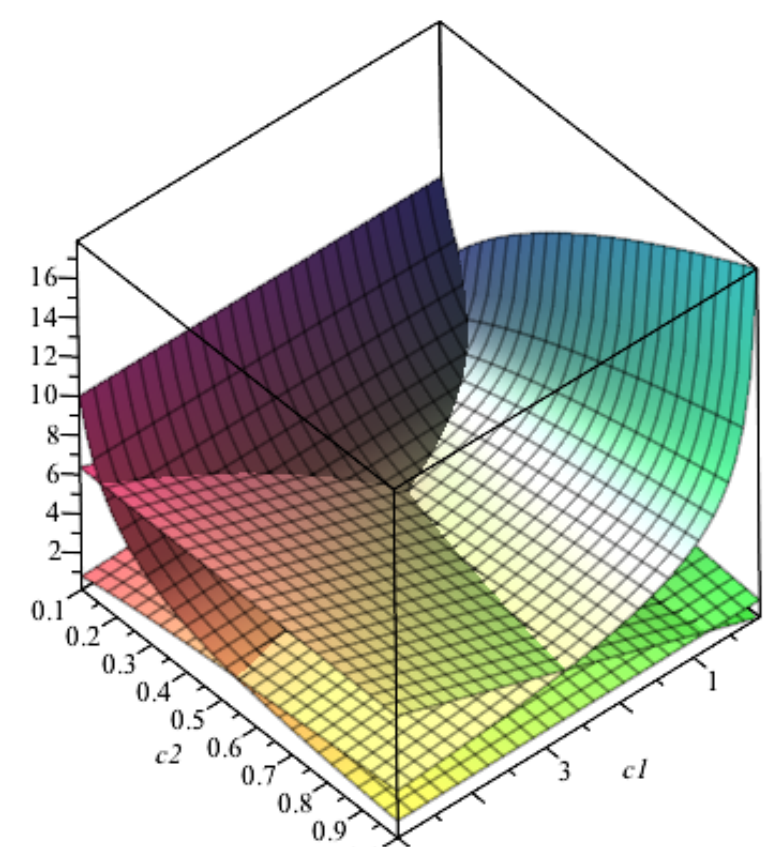

FiguRE 2. The three functions $R_{1}\left(\omega_{1}\right), R_{3}\left(k_{m}\right)$ and $R_{3}\left(k_{\max }\right)$ with the minimizing point in the middle.

Hence the asymptotic solution of the min-max problem (10) for $\alpha>\beta$ is

$$
\begin{gathered}
C_{1}^{*}=\left(\frac{r}{2}\right)^{\frac{1}{4}}\left(\frac{c_{\max }}{h}\right)^{\frac{3}{4}}, \quad C_{2}^{*}=\frac{1}{2}\left(\frac{r}{2}\right)^{\frac{3}{4}}\left(\frac{c_{\max }}{h}\right)^{\frac{1}{4}}, \\
\rho_{\mathrm{opt}}^{*}=1-\left(\frac{r}{2 c_{\max }}\right)^{\frac{1}{4}} h^{\frac{1}{4}}+\mathcal{O}\left(h^{\frac{1}{2}}\right) .
\end{gathered}
$$

Case II $(\beta=\alpha)$ : In this case we have $C_{1}=\frac{c_{1}}{h^{\alpha}}$ and $C_{2}=\frac{c_{2}}{h^{\alpha}}$ and we follow the same steps as in the case $\alpha>\beta$.

1. For the first interval $\left[0, \omega_{1}\right]$ we obtain from $(21)$ for $\alpha=\beta$

$$
\frac{d R_{1}}{d k}\left(k, \omega_{1}, \omega_{2}, C_{1}, C_{2}\right)=k\left[\left(\frac{1}{c_{1}}+\frac{1}{c_{2}}\right)\left(\frac{1}{\tilde{\lambda}_{1}}+\frac{1}{\tilde{\lambda}_{2}}\right) h^{\alpha}+\mathcal{O}\left(h^{2 \alpha}\right)\right]
$$

which shows that the only extremum at $k=0$ is a minimum. For the second interval $\left[\omega_{1}, \omega_{2}\right]$ we get from $(22)$ for $\alpha=\beta$

$$
\frac{d R_{2}}{d k}\left(k, \omega_{1}, \omega_{2}, C_{1}, C_{2}\right)=k\left[\left(\frac{1}{c_{1}}+\frac{1}{c_{2}}\right)\left(\frac{\lambda_{1}-\tilde{\lambda}_{2}}{\lambda_{1} \tilde{\lambda}_{2}}\right) h^{\alpha}+\mathcal{O}\left(h^{2 \alpha}\right)\right] .
$$

This shows that we have a local extremum if $\lambda_{1}=\tilde{\lambda}_{2}$, i.e. $k=k_{1}:=\frac{\omega_{1}^{2}+\omega_{2}^{2}}{2}$, and a further study of the signs reveals that it is a local minimum. For the third interval $\left[\omega_{2}, k_{\max }\right]$ we get from (23)

$$
\frac{d R_{3}}{d k}\left(k, \omega_{1}, \omega_{2}, C_{1}, C_{2}\right)=-k\left[\left(\frac{1}{c_{1}}+\frac{1}{c_{2}}\right)\left(\frac{\lambda_{1}+\lambda_{2}}{\lambda_{1} \lambda_{2}}\right) h^{\alpha}+\mathcal{O}\left(h^{2 \alpha}\right)\right]
$$

which shows that $R_{3}$ is decreasing in the third interval. 
2. For extrema close to 0 we set $k=c_{m} h^{\gamma}$, with $\gamma>0$, and get from $(24)$

$$
\frac{d R_{1}}{d k}\left(k, \omega_{1}, \omega_{2}, C_{1}, C_{2}\right)=c_{m}\left(\frac{1}{\omega_{1}}+\frac{1}{\omega_{2}}\right)\left(\frac{1}{c_{1}}+\frac{1}{c_{2}}\right) h^{\alpha-\gamma}+\mathcal{O}\left(h^{\min \{\alpha+\gamma, 2 \alpha\}}\right),
$$

which shows that $R_{1}$ is asymptotically increasing close to 0 .

3. Now we classify the extrema of the form $k=c_{m} / h^{\gamma}$, with $0<\gamma \leq 1$. Here, we only have 3 cases $(\gamma<\alpha$, $\gamma=\alpha$ and $\alpha<\gamma$ ). For $\gamma<\alpha$, we obtain from (25)

$$
\frac{d R_{3}}{d k}\left(k, \omega_{1}, \omega_{2}, C_{1}, C_{2}\right)=-2\left(\frac{1}{c_{1}}+\frac{1}{c_{2}}\right) h^{\alpha}+\mathcal{O}\left(h^{\min \{2 \alpha-\gamma, \alpha+2 \gamma\}}\right),
$$

and thus $R_{3}$ is decreasing. For $\alpha<\gamma$, using (26) we obtain

$$
\frac{d R_{3}}{d k}\left(k \omega_{1}, \omega_{2}, C_{1}, C_{2}\right)=\left(\frac{4}{c_{m}^{2}}\right)\left(c_{1}+c_{2}\right) h^{\gamma-\alpha}+\mathcal{O}\left(h^{3 \gamma-2 \alpha}\right),
$$

and therefore $R_{3}$ is increasing. Finally for $\alpha=\gamma$, we perform the calculations as in the case $\left[0, \omega_{1}\right]$ for $\alpha>\beta$ (for details see [22]), and obtain

$$
\frac{d R_{3}}{d k}(k)=\frac{4\left(c_{1}+c_{2}\right)\left(c_{m}^{2}-2 c_{1} c_{2}\right)\left(c_{m}^{4}-2 c_{m}^{2}\left(c_{1}-c_{2}\right)^{2}+4 c_{1}^{2} c_{2}^{2}\right)}{\left(c_{m}^{2}+2 c_{m} c_{1}+2 c_{1}^{2}\right)^{2}\left(c_{m}^{2}+2 c_{m} c_{2}+2 c_{2}^{2}\right)^{2}} h^{\alpha}+\mathcal{O}\left(h^{*}\right),
$$

with * a term greater than $\alpha$. The leading order term of (33) thus vanishes for three positive values of $c_{m}$, namely

$$
\begin{aligned}
& c_{m_{1}}=\sqrt{\left(c_{1}-c_{2}\right)^{2}-\sqrt{\left(c_{1}^{2}+c_{2}^{2}\right)\left(c_{1}^{2}-4 c_{1} c_{2}+c_{2}^{2}\right)}}, \\
& c_{m_{2}}=\sqrt{2 c_{1} c_{2}}, \\
& c_{m_{3}}=\sqrt{\left(c_{1}-c_{2}\right)^{2}+\sqrt{\left(c_{1}^{2}+c_{2}^{2}\right)\left(c_{1}^{2}-4 c_{1} c_{2}+c_{2}^{2}\right)}} .
\end{aligned}
$$

One can verify that these solutions are in increasing order if they are real (which means that $c_{1}^{2}-4 c_{1} c_{2}+c_{2}^{2} \geq 0$ ). A sign study of (33) implies that we have a maximum at $k=k_{m}:=\frac{c_{m_{2}}}{h^{\alpha}}=\frac{\sqrt{2 c_{1} c_{2}}}{h^{\alpha}}$ when $c_{m_{1}}$ and $c_{m_{3}}$ are real and only a minimum at $k=k_{m}$ when $c_{m_{1}}$ and $c_{m_{3}}$ are complex.

4. From the previous analysis we know that the candidates for the optimization are $k=\omega_{1}, k=k_{m}=\frac{\sqrt{2 c_{1} c_{2}}}{h^{\alpha}}$ and $k=k_{\max }=\frac{c_{\max }}{h}$. From (28) for the case $\alpha=\beta$ we get

$$
R_{1}\left(\omega_{1}, \omega_{1}, \omega_{2}, C_{1}, C_{2}\right)=1-r\left(\frac{1}{c_{1}}+\frac{1}{c_{2}}\right) h^{\alpha}+\mathcal{O}\left(h^{2 \alpha}\right) .
$$

Similarly from (31), we have

$$
R_{3}\left(k_{\max }, \omega_{1}, \omega_{2}, C_{1}, C_{2}\right)=1-\frac{4}{c_{\max }}\left(c_{1}+c_{2}\right) h^{1-\alpha}+\mathcal{O}\left(h^{2-2 \alpha}\right)
$$

For $k_{m}=\frac{\sqrt{2 c_{1} c_{2}}}{h^{\alpha}}$, we use the asymptotic expansions we computed, and obtain after simplifying

$$
R_{3}\left(k_{m}, \omega_{1}, \omega_{2}, C_{1}, C_{2}\right)=\left(\frac{\left(c_{1}-\sqrt{2 c_{1} c_{2}}+c_{2}\right)^{2}}{\left(c_{1}+\sqrt{2 c_{1} c_{2}}+c_{2}\right)^{2}}\right)\left(1+\mathcal{O}\left(h^{2 \alpha}\right)\right)
$$

The leading order term of (36) does not dependent on $h$, and it is not difficult to verify that it is smaller than 1 for any $c_{1}, c_{2}>0$. Then the only candidates for the optimization are (34) and (35). Comparing equations (34) 

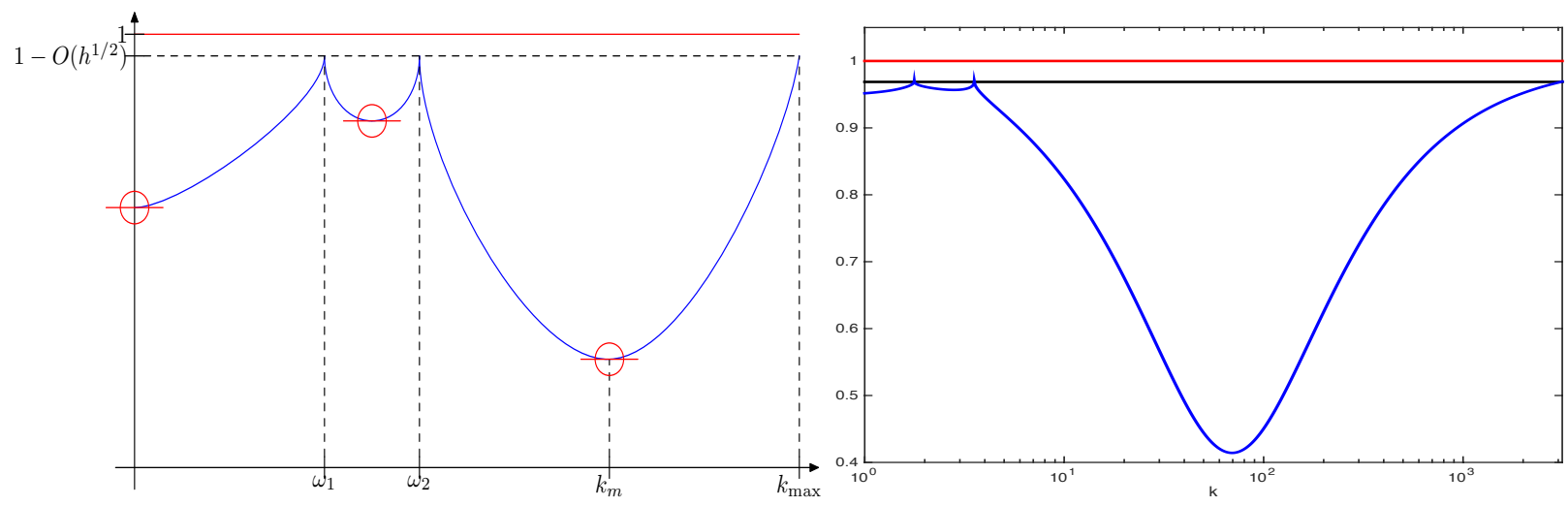

FiguRE 3. Left: drawing of the optimized convergence factor $\rho_{\text {opt }}$ studied in Theorem 3 for the case $\alpha=\beta$. Right: actual plot of the optimized convergence factor from Theorem 3 for $\mu_{1}=\mu_{2}=1, \varepsilon_{1}=1, \varepsilon_{2}=4, \omega=\pi$ and $h=10^{-3}$. The red line is at 1 and the black line is the asymptotic maximum $\left(1-\mathcal{O}\left(h^{\frac{1}{2}}\right)\right.$ for this case).

and (35) we see that $\alpha$ and $1-\alpha$ are in competition and we thus have to equilibrate them (i.e. $\alpha=1-\alpha$ which implies $\alpha=1 / 2)$. This gives the order of the convergence factor $\rho^{*}=1-\mathcal{O}\left(h^{\frac{1}{2}}\right)$ which is worse than Case I $(\beta<\alpha)$ studied before. So we can exclude this case as solution of the optimization problem.

Case III $(\beta>\alpha)$ : This case is completely symmetric to $\beta<\alpha$, just the roles of $c_{1}$ and $c_{2}$ and the roles of $\alpha$ and $\beta$ are exchanged. The solution then gives the second part of the theorem.

\section{Transmission CONDItions FOR THE TEz MODE}

We now study transmission conditions for the TEz mode for the physically important case $\mu_{1}=\mu_{2}$ and $\varepsilon_{1} \neq \varepsilon_{2}$. Using Remark 1, one can also obtain an equivalent result for the TEz mode for the case $\mu_{1} \neq \mu_{2}$ and $\varepsilon_{1}=\varepsilon_{2}$, which was announced without proof and in less general form in [20], and used in [21] to obtain results for the $3 \mathrm{~d}$ case.

Theorem 5. If $\mu_{1}=\mu_{2}, \varepsilon_{1} \neq \varepsilon_{2}, s_{1}=(1+i) C_{1}, s_{2}=(1+i) C_{2}, r=\sqrt{\left|\omega_{2}^{2}-\omega_{1}^{2}\right|}, \varepsilon=\sqrt{\varepsilon_{1} / \varepsilon_{2}}$ and $c_{\mathrm{max}}$ is given by the relationship $k_{\max }=\frac{c_{\max }}{h}$, we have the following results:

- If $0<\varepsilon^{2} \leq \frac{1}{2}$, then the asymptotic solution of the min-max problem (12) for $h$ small is given by

$$
C_{1}^{*} \geq \frac{c_{\max } \varepsilon(\sqrt{3+4 \varepsilon}-1-2 \varepsilon)}{2 h\left(1-2 \varepsilon^{2}\right)}, \quad C_{2}^{*}=r, \quad \rho_{o p t}^{*}=\sqrt[4]{\frac{1}{2}}+\mathcal{O}(h)
$$

- If $\frac{1}{2}<\varepsilon^{2}<1$, then the asymptotic solution of the min-max problem (12) for $h$ small is given by

$$
C_{1}^{*} \geq \frac{c_{\max }(1-\varepsilon)}{2 h}, \quad \frac{r}{1+\sqrt{2 \varepsilon^{2}-1}} \leq C_{2}^{*} \leq \frac{r}{1-\sqrt{2 \varepsilon^{2}-1}}, \quad \rho_{o p t}^{*}=\sqrt{\varepsilon}+\mathcal{O}(h)
$$

- If $1<\varepsilon^{2} \leq 2$, then the asymptotic solution of the min-max problem (12) for $h$ small is given by

$$
\frac{r \varepsilon}{\varepsilon+\sqrt{2-\varepsilon^{2}}} \leq C_{1}^{*} \leq \frac{r \varepsilon}{\varepsilon-\sqrt{2-\varepsilon^{2}}}, \quad C_{2}^{*} \geq \frac{c_{\max }(\varepsilon-1)}{2 h \varepsilon}, \quad \rho_{o p t}^{*}=\frac{1}{\sqrt{\varepsilon}}+\mathcal{O}(h)
$$

- If $\varepsilon^{2} \geq 2$, then the asymptotic solution of the min-max problem (12) for $h$ small is given by

$$
C_{1}^{*}=r, \quad C_{2}^{*} \geq \frac{c_{\max }}{2 h} \frac{\sqrt{3 \varepsilon^{2}+4 \varepsilon}-\varepsilon-2}{\varepsilon^{2}-2}, \quad \rho_{o p t}^{*}=\sqrt[4]{\frac{1}{2}}+\mathcal{O}(h)
$$


Remark 6. A more general form of Theorem 5 is presented in [22] for the TMz case with $\mu_{1} \neq \mu_{2}$ and $\omega_{1} \neq \omega_{2}$ which guarantees convergence independently of the mesh size $h$. Similarly, there is a more general form of Theorem 5 for $\varepsilon_{1} \neq \varepsilon_{2}$ and $\omega_{1} \neq \omega_{2}$ that guarantees also convergence independently of the mesh size $h$. Combining both results we have (for $\mu_{1} \neq \mu_{2}, \varepsilon_{1} \neq \varepsilon_{2}$ and $\omega_{1} \neq \omega_{2}$ ) a non-overlapping Optimized Schwarz Method applied to the complete Maxwell system in $3 d$ that converges independently of the mesh size $h$. The condition $\mu_{1} \neq \mu_{2}, \varepsilon_{1} \neq \varepsilon_{2}$ and $\omega_{1} \neq \omega_{2}$ is usually verified if we consider two different materials, nevertheless for the case $\mu_{1}=\mu_{2}$ and $\varepsilon_{1} \neq \varepsilon_{2}$ the complete Maxwell system in $3 d$ has a contraction factor of $1-\mathcal{O}\left(h^{\frac{1}{4}}\right)$, as in the continuous case studied in [8].

Proof. We proceed as in the proof of Theorem 3: we use the ansatz $C_{1}:=\frac{c_{1}}{h^{\alpha}}$ and $C_{2}:=\frac{c_{2}}{h^{\beta}}$, divide the proof into Case I: $\beta<\alpha$, Case II: $\beta=\alpha$, and Case III: $\beta>\alpha$, and perform the four steps to identify and balance maxima.

Case I $(\beta<\alpha)$ :

1. First we show asymptotically that there is only one local extremum for $k=c$ constant, proceeding as in Theorem 3 for the case $\alpha>\beta$, for details see [22]. We obtain for the derivative for $k \in\left(0, \omega_{1}\right)$

$$
\frac{d R_{1}}{d k}\left(k, \omega_{1}, \omega_{2}, \varepsilon, C_{1}, C_{2}\right)=k\left(\frac{1}{\tilde{\lambda}_{1}}+\frac{\varepsilon}{\tilde{\lambda}_{2}}\right)\left[\frac{1}{\varepsilon c_{2}} h^{\beta}+\frac{1}{c_{1}} h^{\alpha}+\mathcal{O}\left(h^{2 \beta}\right)\right] .
$$

We thus have a local extremum for $k=0$, and $\frac{d R_{1}}{d k}>0, \forall k \in\left(0, \omega_{1}\right)$. So there are no other local extrema for fixed $k$, and $R_{1}$ is increasing in $\left(0, \omega_{1}\right)$, which implies that $k=0$ is a minimum, and the maximum is at $k=\omega_{1}$. For $k \in\left(\omega_{1}, \omega_{2}\right)$ we get

$$
\frac{d R_{2}}{d k}\left(k, \omega_{1}, \omega_{2}, C_{1}, C_{2}\right)=k\left(\frac{\lambda_{1} \varepsilon-\tilde{\lambda}_{2}}{\lambda_{1} \tilde{\lambda}_{2}}\right)\left[\frac{1}{\varepsilon c_{2}} h^{\beta}+\frac{1}{c_{1}} h^{\alpha}+\mathcal{O}\left(h^{2 \beta}\right)\right] .
$$

Hence the leading order term vanishes if $\tilde{\lambda}_{2}=\lambda_{1} \varepsilon$, which means $k=\sqrt{\frac{\omega_{1}^{2} \varepsilon^{2}+\omega_{2}^{2}}{1+\varepsilon^{2}}}$. If $k<\sqrt{\frac{\omega_{1}^{2} \varepsilon^{2}+\omega_{2}^{2}}{1+\varepsilon^{2}}}$ we have $\lambda_{1} \varepsilon<\tilde{\lambda}_{2}$ and thus $\frac{d R_{2}}{d k}<0$. If $k>\sqrt{\frac{\omega_{1}^{2} \varepsilon^{2}+\omega_{2}^{2}}{1+\varepsilon^{2}}}$ we have $\lambda_{1} \varepsilon>\tilde{\lambda}_{2}$ which gives $\frac{d R_{2}}{d k}>0$. These three conditions imply that we have a local minimum. The maximum of the interval is therefore necessarily either at $k=\omega_{1}$ or $k=\omega_{2}$. For $k \in\left(\omega_{2}, k_{\max }\right)$, we finally get

$$
\frac{d R_{3}}{d k}\left(k, \omega_{1}, \omega_{2}, \varepsilon, C_{1}, C_{2}\right)=-k\left(\frac{\varepsilon \lambda_{1}+\lambda_{2}}{\lambda_{1} \lambda_{2}}\right)\left[\frac{1}{\varepsilon c_{2}} h^{\beta}+\frac{1}{c_{1}} h^{\alpha}+\mathcal{O}\left(h^{2 \beta}\right)\right] .
$$

Since $\frac{\lambda_{1} \varepsilon+\lambda_{2}}{\lambda_{1} \lambda_{2}}>0$ for $k \in\left(\omega_{2}, k_{\max }\right)$ we deduce that $R_{3}$ does not have extrema for fixed $k$ in $\left[\omega_{2}, k_{\max }\right]$, and the sign of the derivative shows that $R_{3}$ is decreasing for $k>\omega_{2}$. Therefore, for fixed $k$ in $\left[0, k_{\max }\right]$, the maxima of $\rho_{\text {opt }}$ are at $k=\omega_{1}$ and $k=\omega_{2}$, for an illustration, see Figure 4 and 5 .

2. Now we show that there is no other extremum close to $k=0$. Proceeding as in the proof of Theorem 3 , we set $k=c_{m} h^{\gamma}$, with $\gamma>0$ and obtain after some computations (for details see [22])

$$
\frac{d R_{1}}{d k}\left(c_{m} h^{\gamma}, \omega_{1}, \omega_{2}, \varepsilon, C_{1}, C_{2}\right)=\left(\frac{c_{m}\left(\omega_{1}+\varepsilon \omega_{2}\right)}{\omega_{1} \omega_{2}} h^{\gamma}\right)\left[\frac{h^{\beta}}{\varepsilon c_{2}}+\frac{h^{\alpha}}{c_{1}}+\mathcal{O}\left(h^{*}\right)\right]
$$

with $*$ a term bigger than $\gamma$. Clearly the leading order term can not vanish for $c_{m}>0$ and $\gamma>0$, so we do not have an extremum close to $k=0$.

3. Proceeding as in the proof of Theorem 3, we now study possible extrema for $k=c_{m} / h^{\gamma}$, with $0<\gamma \leq 1$. We have to consider 5 sub-cases: $\gamma<\beta<\alpha, \beta<\gamma<\alpha, \beta<\alpha<\gamma$, and the two particular cases $\beta=\gamma<\alpha$ 

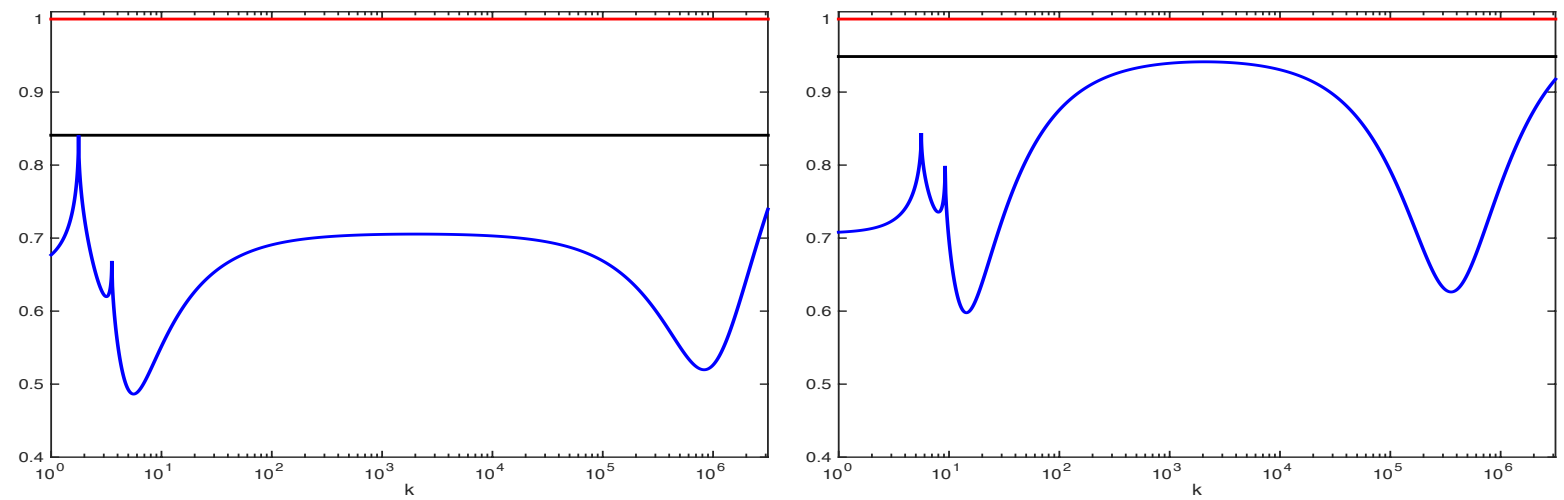

FIGURE 4. Convergence factor $\rho_{\text {opt }}$ from the first and the second case of Theorem $5\left(\varepsilon_{1}<\varepsilon_{2}\right)$. The red line denotes 1 , the black line is the asymptotic maximum: $\sqrt[4]{\frac{1}{2}}$ for the left case with $\mu_{1}=\varepsilon_{1}=\mu_{2}=1, \varepsilon_{2}=2, \omega=\pi$ and $h=10^{-6}$, and $\varepsilon$ for the right case with $\varepsilon_{1}=9$, $\varepsilon_{2}=\mu_{1}=\mu_{2}=10, \omega=\pi$ and $h=10^{-6}$. Convergence does not depend on the mesh size $h$.
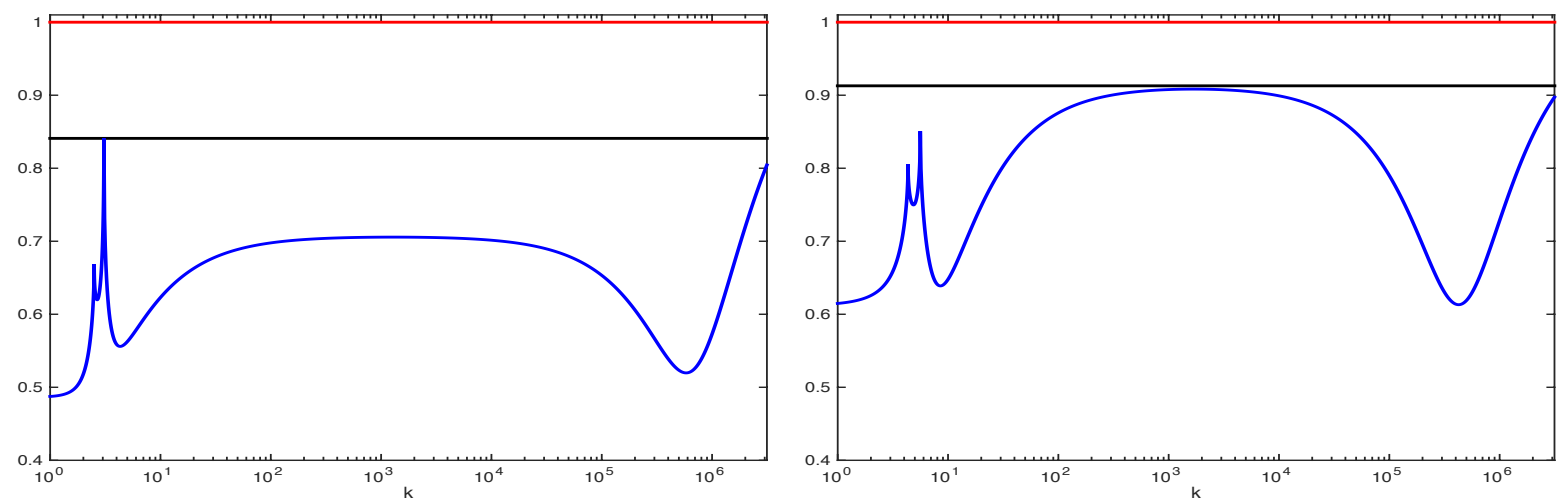

Figure 5. Convergence factor $\rho_{\text {opt }}$ from the third and fourth case of Theorem $5\left(\varepsilon_{2}<\varepsilon_{1}\right)$. The red line is 1 , the black line is the asymptotic maximum: $\sqrt[4]{\frac{1}{2}}$ for the left case with $\mu_{1}=$ $\varepsilon_{2}=\mu_{2}=1, \varepsilon_{1}=2, \omega=\pi$ and $h=10^{-6}$ and $\varepsilon$ for the right case with $\varepsilon_{1}=6, \varepsilon_{2}=5, \mu_{1}=1$, $\mu_{2}=1, \omega=\pi$ and $h=10^{-6}$. Convergence does not depend on the mesh size $h$.

and $\beta<\gamma=\alpha$. In order to simplify the notation we use again $R_{3}(k)$ to denote $R_{3}\left(k, \omega_{1}, \omega_{2}, \varepsilon, C_{1}, C_{2}\right)$. For the case $\gamma<\beta<\alpha$, we obtain for the derivative

$$
\frac{d R_{3}}{d k}(k)=-\frac{(1+\varepsilon)}{\varepsilon c_{2}} h^{\beta}-\frac{(1+\varepsilon)}{c_{1}} h^{\alpha}+\mathcal{O}\left(h^{\min \{\beta+2 \gamma, 2 \beta-\gamma,\}}\right),
$$

which shows that the leading order term can not vanish for a particular choice of $\gamma$ and $c_{m}$, since they are not present in the leading order term. Hence there is no local extremum for $\gamma<\beta<\alpha$. For the case $\beta<\alpha<\gamma$, we get

$$
\frac{d R_{3}}{d k}(k)=\left(\frac{2(1+\varepsilon)}{c_{m}^{2}} h^{\gamma}\right)\left(\frac{c_{1}}{\varepsilon} h^{\gamma-\alpha}+c_{2} h^{\gamma-\beta}+\mathcal{O}\left(h^{2 \gamma-2 \alpha}\right)\right)
$$


Again the leading order term can not vanish, and hence there is no local extremum for $k$ of the form $k=c_{m} / h^{\gamma}$ and $\beta<\alpha<\gamma$. We further see that $R_{3}$ is increasing in this case. For the case $\beta<\gamma<\alpha$, we find

$$
\frac{d R_{3}}{d k}(k)=\frac{2 \varepsilon^{2}(1+\varepsilon) c_{2}}{c_{m}^{2}} h^{2 \gamma-\beta}-\frac{\varepsilon^{2}(1+\varepsilon)}{c_{1}} h^{\alpha}+\mathcal{O}\left(h^{*}\right),
$$

with $*=\min \{2 \alpha-\gamma, \alpha+\gamma-\beta, 3 \gamma-2 \beta\}$. The leading order term can thus vanish if we have $\gamma=(\alpha+\beta) / 2$, and rewriting (47) for this choice, we obtain

$$
\frac{d R_{3}}{d k}(k)=\frac{\varepsilon^{2}(1+\varepsilon)\left(2 c_{1} c_{2}-c_{m}^{2}\right)}{c_{m}^{2} c_{1}} h^{\alpha}+\mathcal{O}\left(h^{(3 \alpha-\beta) / 2}\right),
$$

which shows that the leading order term will be zero if $c_{m}=\sqrt{2 c_{1} c_{2}}$. We thus have a local extremum for $\beta<\gamma<\alpha$ and $k=\frac{c_{m}}{h \gamma}$ when $\gamma=(\alpha+\beta) / 2$ and $c_{m}=\sqrt{2 c_{1} c_{2}}$, and it is a maximum after a further study of the signs in (48). For $\beta=\gamma<\alpha$, we get

$$
\frac{d R_{3}}{d k}(k)=\left(\frac{-2 \varepsilon^{2} c_{2}\left(c_{m}^{2}-2\left(c_{m}-c_{2}\right)\left(c_{m}+\varepsilon c_{2}\right)\right)}{\left(\varepsilon^{2} c_{2}^{2}+\left(c_{m}+\varepsilon c_{2}\right)^{2}\right)^{2}}\right) h^{\beta}-\left(\frac{\varepsilon^{2}(1+\varepsilon)\left(c_{2}^{2}+\left(c_{m}-c_{2}\right)^{2}\right)}{c_{1}\left(\varepsilon^{2} c_{2}^{2}+\left(c_{m}+\varepsilon c_{2}\right)^{2}\right)}\right) h^{\alpha}+\mathcal{O}\left(h^{2 \alpha-\beta}\right)
$$

and the leading order term vanishes for $c_{m}=\left(1-\varepsilon+\sqrt{1+\varepsilon^{2}}\right) c_{2}$, which leads however to a local minimum asymptotically at $k=\frac{\left(1-\varepsilon+\sqrt{1+\varepsilon^{2}}\right) c_{2}}{h^{\beta}}$ after a further sign study. Finally we look for extrema when $k=\frac{c_{m}}{h^{\alpha}}$, which leads for the case $\beta<\alpha=\gamma$ to

$$
\frac{d R_{3}}{d k}(k)=\left(\frac{2 \varepsilon^{2} c_{1}(1+\varepsilon)\left(\varepsilon c_{m}^{2}+2 c_{1}(1-\varepsilon) c_{m}-2 c_{1}^{2}\right)}{\left(c_{1}^{2}+\left(\varepsilon c_{m}+c_{1}\right)^{2}\right)^{2}}\right) h^{\alpha}+\mathcal{O}\left(h^{2 \alpha-\beta}\right) .
$$

The leading order term vanishes for $c_{m}=\frac{1-\varepsilon+\sqrt{1+\varepsilon^{2}}}{\varepsilon} c_{1}$, we thus have asymptotically an extremum for $k=$ $\frac{\left(1-\varepsilon+\sqrt{1+\varepsilon^{2}}\right) c_{1}}{\varepsilon h^{\alpha}}$, which turns out however also to be a minimum.

4. We have again identified four candidates for the maximum. We start with the asymptotic expansions of $R_{1}\left(\omega_{1}, \omega_{1}, \omega_{2}, \varepsilon, C_{1}, C_{2}\right)$ and $R_{2}\left(\omega_{2}, \omega_{1}, \omega_{2}, \varepsilon, C_{1}, C_{2}\right)$, and we classify these two expressions as functions of the value of $\varepsilon$. We have

$$
\begin{aligned}
R_{1}\left(\omega_{1}\right) & =\frac{\left(\frac{2 c_{1}^{2}}{h^{2 \alpha}}\right)\left(1-\frac{r}{c_{1}} h^{\alpha}+\mathcal{O}\left(h^{2 \alpha}\right)\right)\left(\frac{2 c_{2}^{2}}{h^{2 \beta}}\right)\left(1-\frac{r}{c_{2}} h^{\beta}+\mathcal{O}\left(h^{2 \beta}\right)\right)}{\left(\frac{2 \varepsilon^{2} c_{2}^{2}}{h^{2 \beta}}\right)\left(1+\frac{r}{\varepsilon c_{2}} h^{\beta}+\mathcal{O}\left(h^{2 \beta}\right)\right)\left(\frac{2 c_{1}^{2}}{\varepsilon^{2}}\right)\left(1+\frac{\varepsilon r}{c_{1}} h^{\alpha}+\mathcal{O}\left(h^{2 \alpha}\right)\right)} \\
& =1-\frac{r}{c_{2}} h^{\beta}-\frac{\varepsilon r}{c_{1}} h^{\alpha}+\mathcal{O}\left(h^{2 \beta}\right),
\end{aligned}
$$

and

$$
\begin{aligned}
R_{2}\left(\omega_{2}\right) & =\frac{\left(\frac{2 c_{1}^{2}}{h^{2 \alpha}}\right)\left(1-\frac{r}{c_{1}} h^{\alpha}+\mathcal{O}\left(h^{2 \alpha}\right)\right)\left(\frac{2 c_{2}^{2}}{h^{2 \beta}}\right)\left(1-\frac{r}{c_{2}} h^{\beta}+\mathcal{O}\left(h^{2 \beta}\right)\right)}{\left(\frac{2 \varepsilon_{2}^{2} c_{2}^{2}}{h^{2 \beta}}\right)\left(1+\frac{r}{\varepsilon c_{2}} h^{\beta}+\mathcal{O}\left(h^{2 \beta}\right)\right)\left(\frac{2 c_{1}^{2}}{\varepsilon^{2} h^{2 \alpha}}\right)\left(1+\frac{r \varepsilon}{c_{1}} h^{\alpha}+\mathcal{O}\left(h^{2 \alpha}\right)\right)} \\
& =1-\frac{r}{\varepsilon c_{2}} h^{\beta}-\frac{r}{c_{1}} h^{\alpha}+\mathcal{O}\left(h^{2 \beta}\right),
\end{aligned}
$$

which shows that $R_{1}\left(\omega_{1}\right)<R_{2}\left(\omega_{2}\right)$ if $\varepsilon>1$ and $R_{1}\left(\omega_{1}\right)>R_{2}\left(\omega_{2}\right)$ if $\varepsilon<1$. Note also that $R_{1}\left(\omega_{1}\right)$ and $R_{2}\left(\omega_{2}\right)$ depend asymptotically on $c_{2}$, and not on $c_{1}$. We next derive the asymptotic expansions of $R_{3}\left(k_{m}, \omega_{1}, \omega_{2}, \varepsilon, C_{1}, C_{2}\right)$ and $R_{3}\left(k_{\max }, \omega_{1}, \omega_{2}, \varepsilon, C_{1}, C_{2}\right)$, and obtain

$$
\begin{aligned}
R_{3}\left(k_{\max }\right) & =\frac{\left(\frac{c_{\max }^{2}}{h^{2}}\right)\left(1-\frac{2 c_{1}}{c_{\max }} h^{1-\alpha}+\mathcal{O}\left(h^{2-2 \alpha}\right)\right)\left(\frac{c_{\max }^{2}}{h^{2}}\right)\left(1-\frac{2 c_{2}}{c_{\max }} h^{1-\beta}+\mathcal{O}\left(h^{2-2 \beta}\right)\right)}{\left(\frac{c_{\max }^{2}}{h^{2}}\right)\left(1+\frac{2 \varepsilon c_{2}}{c_{\max }} h^{1-\beta}+\mathcal{O}\left(h^{2-2 \beta}\right)\right)\left(\frac{c_{\max }^{2}}{h^{2}}\right)\left(1+\frac{2 c_{1}}{\varepsilon c_{\max }} h^{1-\alpha}+\mathcal{O}\left(h^{2-2 \alpha}\right)\right)} \\
& =1-\frac{2 c_{1}}{\varepsilon c_{\max }}(1+\varepsilon) h^{1-\alpha}-\frac{2 \varepsilon c_{2}}{c_{\max }}(1+\varepsilon) h^{1-\beta}+\mathcal{O}\left(h^{2-2 \alpha}\right),
\end{aligned}
$$


and

$$
\begin{aligned}
R_{3}\left(k_{m}\right) & =\frac{\left(\frac{c_{1}^{2}}{h^{2 \alpha}}\right)\left(1-\frac{2 c_{m}}{c_{1}} h^{\frac{\alpha-\beta}{2}}+\mathcal{O}\left(h^{\alpha-\beta}\right)\right)\left(\frac{c_{m}^{2}}{h^{2 \gamma}}\right)\left(1-\frac{2 c_{2}}{c_{m}} h^{\frac{\alpha-\beta}{2}}+\mathcal{O}\left(h^{\alpha-\beta}\right)\right)}{\left(\frac{c_{m}^{2}}{h^{2 \gamma}}\right)\left(1+\frac{2 \varepsilon c_{2}}{c_{m}} h^{\frac{\alpha-\beta}{2}}+\mathcal{O}\left(h^{\alpha-\beta}\right)\right)\left(\frac{c_{1}^{2}}{\varepsilon^{2} h^{2 \alpha}}\right)\left(1+\frac{2 \varepsilon c_{m}}{c_{1}} h^{\frac{\alpha-\beta}{2}}+\mathcal{O}\left(h^{\alpha-\beta}\right)\right)} \\
& =\varepsilon^{2}\left(1-3 \sqrt{2}(1+\varepsilon) \sqrt{\frac{c_{2}}{c_{1}}} h^{\frac{\alpha-\beta}{2}}+\mathcal{O}\left(h^{\alpha-\beta}\right)\right) .
\end{aligned}
$$

We observe from (54) that the leading order term of $R_{3}\left(k_{m}\right)$ does not depend on $\alpha, \beta, c_{1}$ and $c_{2}$, and can thus not be optimized in this case, and additionally $R_{3}\left(k_{m}\right)<1$ if and only if $\varepsilon<1$. We thus consider for the rest of the case $\beta<\alpha$ that we have $\varepsilon<1$ (the case $\varepsilon>1$ will be considered when we study the case $(\alpha<\beta)$ ).

From (51) and (53) we observe that the leading order terms are not dependent on the same variables (i.e. $1-\mathcal{O}\left(h^{\beta}\right)$ and $\left.1-\mathcal{O}\left(h^{1-\alpha}\right)\right)$. This suggest to set $\alpha=1$ and $\beta=0$ to obtain terms independent of the mesh size $h$, since all the other choices will lead to mesh dependence. We thus recompute for $\alpha=1$ and $\beta=0$ the expansions of $R_{1}\left(\omega_{1}\right), R_{3}\left(k_{m}\right)$, and $R_{3}\left(k_{\max }\right)$, and obtain

$$
\begin{aligned}
R_{1}\left(\omega_{1}\right) & =\left(\frac{1}{2} \frac{2 c_{2}^{2}-2 r c_{2}+r^{2}}{c_{2}^{2}}\right)\left(1-\frac{\varepsilon r}{c_{1}} h+\mathcal{O}\left(h^{2}\right)\right) \\
R_{3}\left(k_{m}\right) & =\varepsilon^{2}\left(1-3 \frac{\sqrt{c_{1} c_{2}}(1+\varepsilon) \sqrt{h}}{c_{1}}+\mathcal{O}(h)\right), \\
R_{3}\left(k_{\max }\right) & =\left(\frac{\varepsilon^{2}\left(c_{\max }^{2}-2 c_{1} c_{\max }+2 c_{1}^{2}\right)}{\varepsilon^{2} c_{\max }^{2}+2 c_{1} c_{\max } \varepsilon+2 c_{1}^{2}}\right)\left(1-\frac{2 c_{2}(\varepsilon+1)}{c_{\max }} h+\mathcal{O}\left(h^{2}\right)\right) .
\end{aligned}
$$

Note that the recomputation is really necessary, because the earlier expansions do not hold for $\beta=0$ and $\alpha=1$. Similarly we also check that with $\beta=0$ and $\alpha=1$ we still only have the same candidates for the maximum points as with $0<\beta<\alpha<1$, for more details, see [22].

We see from $(55)$ that $R_{3}\left(k_{m}\right) \sim \varepsilon^{2}$, which can not be influenced any further with the remaining constants $c_{1}$ and $c_{2}$ we can choose. We thus try to minimize the leading order terms of $R_{1}\left(\omega_{1}\right)$ and $R_{3}\left(k_{\max }\right)$ using $c_{2}$ and $c_{1}$. To do so, we check the asymptotic derivatives in $c_{1}$ and $c_{2}$,

$$
\begin{aligned}
\frac{d R_{1}\left(\omega_{1}\right)}{d c_{2}} & \sim \frac{r\left(c_{2}-r\right)}{c_{2}^{3}}, \\
\frac{d R_{3}\left(k_{\max }\right)}{d c_{1}} & \sim-2 \frac{\varepsilon^{2} c_{\max }(\varepsilon+1)\left(c_{\max }^{2} \varepsilon-2 c_{1} c_{\max } \varepsilon+2 c_{1} c_{\max }-2 c_{1}^{2}\right)}{\left(\varepsilon^{2} c_{\max }^{2}+2 c_{1} c_{\max } \varepsilon+2 c_{1}^{2}\right)^{2}} .
\end{aligned}
$$

The unique solution of $\frac{d R_{1}\left(\omega_{1}\right)}{d c_{2}} \sim 0$ is $\tilde{c}_{2}=r$ and this is a minimum because $\frac{d^{2} R_{1}}{d c_{2}^{2}}\left(\omega_{1}, \tilde{c}_{2}\right) \sim \frac{1}{r^{2}}$. We have in this case

$$
R_{1}\left(\omega_{1}, \omega_{1}, \omega_{2}, \varepsilon, C_{1},(1+i) r\right)=\frac{1}{2}+\mathcal{O}(h) .
$$

Similarly the unique positive solution of $\frac{d R_{3}\left(k_{\max }\right)}{d c_{1}} \sim 0$ is $\tilde{c}_{1}:=\frac{c_{\max }}{2}\left(1-\varepsilon+\sqrt{1+\varepsilon^{2}}\right)$, which is also a minimum because second derivative is

$$
\frac{d^{2} R_{3}}{d c_{1}^{2}}\left(k_{\max }, \tilde{c}_{1}\right) \sim 4 \frac{\varepsilon^{2}(\varepsilon+1)\left(\sqrt{\varepsilon^{2}+1} \varepsilon^{2}+\varepsilon^{2}+\sqrt{\varepsilon^{2}+1}+1\right)}{c_{\max }^{2}\left(\varepsilon^{2}+\sqrt{\varepsilon^{2}+1}+1\right)^{3}}>0 .
$$

At this minimum we have

$$
R_{3}\left(k_{\max }, \omega_{1}, \omega_{2}, \varepsilon,(1+i) \tilde{c}_{1} / h, C_{2}\right)=\frac{\varepsilon^{2}\left(\sqrt{1+\varepsilon^{2}}-\varepsilon\right)}{1+\sqrt{1+\varepsilon^{2}}}+\mathcal{O}(h) .
$$

We now verify that for $0<\varepsilon<1$ we have asymptotically $R_{3}\left(k_{\max },(1+i) \tilde{c}_{1} / h\right)<\frac{1}{2}$, which leads for $c_{1}$ to an entire interval in which it can be chosen such that $R_{3}\left(k_{\max }\right)<R_{1}\left(\omega_{1}\right)$, and thus the solution is not unique. To see why $R_{3}\left(k_{\max },(1+i) \tilde{c}_{1} / h\right)<\frac{1}{2}$, we first check that the second factor in the numerator satisfies for $0<\varepsilon<1$ that $0<\sqrt{1+\varepsilon^{2}}-\varepsilon<1$, which can be obtained by noting that $\sqrt{1+\varepsilon^{2}}-\varepsilon$ is a decreasing function 
$\left(\left(\sqrt{1+\varepsilon^{2}}-\varepsilon\right)^{\prime}=\frac{\varepsilon}{\sqrt{1+\varepsilon^{2}}}-1<0\right)$ and its maximum is thus attained at $\varepsilon=0$. We then estimate for the denominator in $R_{3}\left(k_{\max },(1+i) \tilde{c}_{1} / h\right)$ that $\frac{1}{1+\sqrt{2}}<\frac{1}{1+\sqrt{1+\varepsilon^{2}}}<\frac{1}{2}$, which implies $0<R_{3}\left(k_{\max }\right)<\frac{1}{2}$ as claimed.

Since $R_{3}\left(k_{m}\right) \sim \varepsilon^{2}$ which can not be influenced, we can have two possible situations: either $\varepsilon^{2}<1 / 2$ and the convergence speed is limited by $R_{1}\left(\omega_{1}\right)$ at $\rho_{\text {opt }}^{4}=\frac{1}{2}$, or $\varepsilon^{2} \geq 1 / 2$ and the convergence speed is limited by $\rho_{\mathrm{opt}}^{4}=\varepsilon^{2}$ from $R_{3}\left(k_{m}\right)$. In the first case, the asymptotic solutions of the min-max problem (12) for $h$ small are given by (37) in the statement of the theorem.

Now in the second case, $\varepsilon^{2} \geq 1 / 2$, there exist two intervals, one for $c_{1}$ such that $R_{3}\left(k_{\max }, \omega_{1}, \omega_{2}, \varepsilon, C_{1}, C_{2}\right)<\varepsilon^{2}$ and one for $c_{2}$ such that $R_{1}\left(\omega_{1}, \omega_{1}, \omega_{2}, \varepsilon, C_{1}, C_{2}\right)<\varepsilon^{2}$. For $R_{3}\left(k_{\max }\right) \leq \varepsilon^{2}$ asymptotically, $c_{1}$ has to satisfy

$$
\frac{\varepsilon^{2}\left(c_{\max }^{2}-2 c_{1} c_{\max }+2 c_{1}^{2}\right)}{\varepsilon^{2} c_{\max }^{2}+2 c_{1} c_{\max } \varepsilon+2 c_{1}^{2}} \leq \varepsilon^{2} \quad \Longleftrightarrow \quad c_{1} \geq \frac{c_{\max }(1-\varepsilon)}{2},
$$

and for $R_{1}\left(\omega_{1}\right) \leq \varepsilon^{2}$ asymptotically, $c_{2}$ has to satisfy

$$
\frac{2 c_{2}^{2}-2 r c_{2}+r^{2}}{2 c_{2}^{2}} \leq \varepsilon^{2} \quad \Longleftrightarrow \quad \frac{r}{1+\sqrt{2 \varepsilon^{2}-1}} \leq c_{2} \leq \frac{r}{1-\sqrt{2 \varepsilon^{2}-1}}
$$

which leads to (38) in the statement of the theorem.

We now study the Case $\alpha=\beta$ :

1. We proceed as in the case $\beta<\alpha$ : for $k$ constant, independent of $h$ we can adapt the results from (41) to obtain

$$
\frac{d R_{1}}{d k}\left(k, \omega_{1}, \omega_{2}, \varepsilon, C_{1}, C_{2}\right)=k\left(\frac{1}{\tilde{\lambda}_{1}}+\frac{\varepsilon}{\tilde{\lambda}_{2}}\right)\left(\frac{1}{c_{1}}+\frac{1}{\varepsilon c_{2}}\right) h^{\alpha}+\mathcal{O}\left(h^{2 \alpha}\right) .
$$

We see that $R_{1}(k)$ is an increasing function in $\left(0, \omega_{1}\right)$, and it thus has a minimum at $k=0$, and a maximum at $k=\omega_{1}$. Next we adapt the results from $(42)$ for the interval $\left[\omega_{1}, \omega_{2}\right]$ to obtain

$$
\frac{R_{2}}{d k}\left(k, \omega_{1}, \omega_{2}, \varepsilon, C_{1}, C_{2}\right)=k\left(\frac{\lambda_{1} \varepsilon-\tilde{\lambda}_{2}}{\lambda_{1} \tilde{\lambda}_{2}}\right)\left(\frac{1}{c_{1}}+\frac{1}{\varepsilon c_{2}}\right) h^{\alpha}+\mathcal{O}\left(h^{2 \alpha}\right) .
$$

This function vanishes for $\lambda_{1} \varepsilon=\tilde{\lambda}_{2}$, which means for $k=\sqrt{\frac{\omega_{1}^{2} \varepsilon^{2}+\omega_{2}^{2}}{1+\varepsilon^{2}}}$, which turns out to be a local minimum after a sign study. The possible maximum of the interval must hence be at either $k=\omega_{1}$ or $k=\omega_{2}$. For the third interval $\left[\omega_{2}, k_{\max }\right]$ we can adapt the results in (43) to obtain

$$
\frac{d R_{3}}{d k}\left(k, \omega_{1}, \omega_{2}, \varepsilon, C_{1}, C_{2}\right)=-k\left(\frac{1}{\lambda_{1}}+\frac{\varepsilon}{\lambda_{2}}\right)\left(\frac{1}{c_{1}}+\frac{1}{\varepsilon c_{2}}\right) h^{\alpha}+\mathcal{O}\left(h^{2 \alpha}\right) .
$$

This shows that $R_{3}$ can not have a local extremum asymptotically for fixed $k$ independent of $h$ and $k \geq \omega_{2}$.

2. Now we consider $k=c_{m} h^{\gamma}$ with $\gamma>0$, and adapting (44) leads to

$$
\frac{d R_{1}}{d k}\left(c_{m} h^{\gamma}, \omega_{1}, \omega_{2}, \varepsilon, C_{1}, C_{2}\right)=c_{m}\left(\frac{1}{\omega_{2}}+\frac{\varepsilon}{\omega_{1}}\right)\left(\frac{1}{c_{1}}+\frac{1}{\varepsilon c_{2}}\right) h^{\alpha+\gamma}+\mathcal{O}\left(h^{*}\right)
$$

with $*=\min \{2 \alpha+\gamma, 3 \gamma+\alpha\}$. The leading term can thus not vanish for any choice of $c_{m}$ or $\gamma$ either, and hence we do not have extrema dependent on $h$ close to 0 .

3. Now we have to study the situation when $k=\frac{c_{m}}{h \gamma}$. For $\alpha=\beta$ we only have three cases to consider: $\gamma<\alpha$, $\gamma=\alpha$ and $\gamma>\alpha$. For $\gamma<\alpha$ we can adapt (45) to get

$$
\frac{d R_{3}}{d k}\left(k, \omega_{1}, \omega_{2}, \varepsilon, C_{1}, C_{2}\right)=-(1+\varepsilon)\left(\frac{1}{c_{1}}+\frac{1}{\varepsilon c_{2}}\right) h^{\alpha}+\mathcal{O}\left(h^{\min \{\alpha+2 \gamma, 2 \gamma-\alpha\}}\right) .
$$


This shows that $R_{3}(k)$ is a decreasing function for $\gamma<\alpha$ and we do not have a local extremum. For $\alpha<\gamma$ we can also adapt (46) to obtain

$$
\frac{d R_{3}}{d k}\left(k, \omega_{1}, \omega_{2}, \varepsilon, C_{1}, C_{2}\right)=\left(\frac{2(1+\varepsilon)}{c_{m}^{2}}\right)\left(\frac{c_{1}}{\varepsilon}+c_{2}\right) h^{2 \gamma-\alpha}+\mathcal{O}\left(h^{3 \gamma-2 \alpha}\right) .
$$

Hence $R_{3}(k)$ is now an increasing function and we do not have an extremum either. The interesting case is $\gamma=\alpha$ : here we have to recompute the asymptotic terms as we did in Theorem 3 in the case $\alpha>\beta$, see [22] for details, and obtain

$$
\begin{aligned}
\frac{d R_{3}}{d k}(k)= & {\left[\frac{2 \varepsilon^{2} c_{m}(1+\varepsilon)\left(c_{1}+\varepsilon c_{2}\right)\left(c_{m}^{2}-2 c_{1} c_{2}\right)}{\left(\varepsilon^{2} c_{2}^{2}+\left(c_{m}+\varepsilon c_{2}\right)^{2}\right)\left(c_{1}^{2}+\left(\varepsilon c_{m}+c_{1}\right)^{2}\right)}\right] } \\
& \cdot\left[\varepsilon c_{m}^{4}-2\left(c_{1}+c_{2}\right)\left(c_{1}+\varepsilon^{2} c_{2}\right) c_{m}^{3}+\left[-2(\varepsilon-1)\left(c_{1}-\varepsilon c_{2}\right)+\right.\right. \\
& \left.\left.+8 \varepsilon c_{1} c_{2}\right] c_{m}^{2}-4 c_{1} c_{2}(\varepsilon-1)\left(c_{1}-\varepsilon c_{2}\right) c_{m}+4 \varepsilon c_{1}^{2} c_{2}^{2}\right]+\mathcal{O}\left(h^{2 \alpha}\right)
\end{aligned}
$$

The positive solutions for a vanishing leading order term are

$$
\begin{aligned}
c_{m_{2}}= & \sqrt{2 c_{1} c_{2}} \\
c_{m_{1,3}}= & \frac{1}{2 \varepsilon}\left[(\varepsilon-1)\left(c_{1}-\varepsilon c_{2}\right)+\sqrt{\left(\varepsilon^{2}+1\right)\left(c_{1}^{2}+\varepsilon^{2} c_{2}^{2}\right)} \pm\right. \\
& \left. \pm\left(\left((\varepsilon-1)\left(c_{1}-\varepsilon c_{2}\right)+\sqrt{\left(\varepsilon^{2}+1\right)\left(c_{1}^{2}+\varepsilon^{2} c_{2}^{2}\right)}\right)^{2}-8 \varepsilon^{2} c_{1} c_{2}\right)^{\frac{1}{2}}\right] .
\end{aligned}
$$

To show that the first solution $c_{m_{2}}=\sqrt{2 c_{1} c_{2}}$ lies between $c_{m_{1}}$ and $c_{m_{3}}$ if they are real, we define

$$
\begin{aligned}
a & :=(\varepsilon-1)\left(c_{1}-\varepsilon c_{2}\right)+\sqrt{\left(\varepsilon^{2}+1\right)\left(c_{1}^{2}+\varepsilon^{2} c_{2}^{2}\right)}, \\
b & :=\sqrt{2 c_{1} c_{2}} .
\end{aligned}
$$

To have $c_{m_{1}}$ and $c_{m_{3}}$ real we need $a \geq 2 \varepsilon b$. We then rewrite the inequality $c_{m_{1}} \leq c_{m_{2}} \leq c_{m_{3}}$ as

$$
\frac{a-\sqrt{a^{2}-4 \varepsilon^{2} b^{2}}}{2 \varepsilon} \leq b \leq \frac{a+\sqrt{a^{2}-4 \varepsilon^{2} b^{2}}}{2 \varepsilon} .
$$

The first inequality follows from $\frac{a-\sqrt{a^{2}-4 \varepsilon^{2} b^{2}}}{2 \varepsilon}=\frac{4 \varepsilon^{2} b^{2}}{2 \varepsilon\left(a+\sqrt{a^{2}-4 \varepsilon^{2} b^{2}}\right)}$ and using that $a \geq 2 \varepsilon b$, and the second inequality holds because $2 \varepsilon b-a \leq 0 \leq \sqrt{a^{2}-4 \varepsilon^{2} b^{2}}$. Hence $c_{m}=\sqrt{2 c_{1} c_{2}}$ is a maximum and the other two are minima in the case when they are real. We denote by $k_{m}:=\sqrt{\frac{2 c_{1} c_{2}}{h}}$ this maximum point. This leads to the asymptotic value

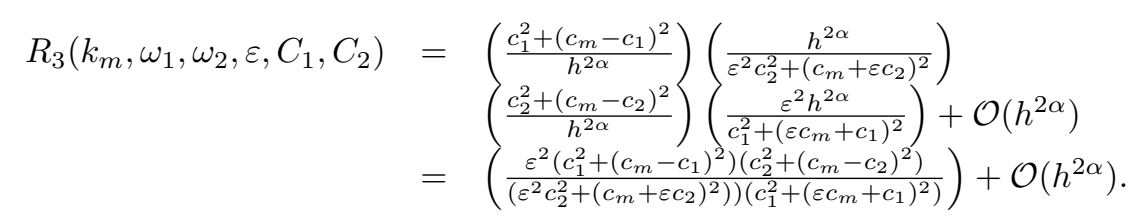

We have therefore identified the possible maxima: $R_{1}\left(\omega_{1}\right), R_{2}\left(\omega_{2}\right), R_{3}\left(k_{m}\right)$ and $R_{3}\left(k_{\max }\right)$, which are asymptotically given by

$$
\begin{aligned}
R_{1}\left(\omega_{1}, \omega_{1}, \omega_{2}, \varepsilon, C_{1}, C_{2}\right) & =1-r\left(\frac{1}{c_{2}}+\frac{\varepsilon}{c_{1}}\right) h^{\alpha}+\mathcal{O}\left(h^{2 \alpha}\right) \\
R_{2}\left(\omega_{2}, \omega_{1}, \omega_{2}, \varepsilon, C_{1}, C_{2}\right) & =1-\frac{r}{\varepsilon}\left(\frac{1}{c_{2}}+\frac{\varepsilon}{c_{1}}\right) h^{\alpha}+\mathcal{O}\left(h^{2 \alpha}\right) \\
R_{3}\left(k_{\max }, \omega_{1}, \omega_{2}, \varepsilon, C_{1}, C_{2}\right) & =1-\frac{2(1+\varepsilon)}{c_{m}}\left(\frac{c_{1}}{\varepsilon}+c_{2}\right) h^{1-\alpha}+\mathcal{O}\left(h^{2-2 \alpha}\right) \\
R_{3}\left(k_{m}, \omega_{1}, \omega_{2}, \varepsilon, C_{1}, C_{2}\right) & =\left(\frac{\varepsilon^{2}\left(c_{1}^{2}+\left(c_{m}-c_{1}\right)^{2}\right)\left(c_{2}^{2}+\left(c_{m}-c_{2}\right)^{2}\right)}{\left.\left(\varepsilon^{2} c_{2}^{2}+\left(c_{m}+\varepsilon c_{2}\right)^{2}\right)\right)\left(c_{1}^{2}+\left(\varepsilon c_{m}+c_{1}\right)^{2}\right)}\right)+\mathcal{O}\left(h^{2 \alpha}\right) .
\end{aligned}
$$




\begin{tabular}{|c|c|c|c|c|c|c|}
\hline$h$ & $\frac{1}{8}$ & $\frac{1}{16}$ & $\frac{1}{32}$ & $\frac{1}{64}$ & $\frac{1}{128}$ & $\frac{1}{256}$ \\
\hline Theorem 3 & 24 & 25 & 30 & 34 & 40 & 46 \\
\hline Theorem 5(Case 1) & 28 & 28 & 30 & 30 & 30 & 30 \\
\hline Theorem 5(Case 2) & 25 & 31 & 31 & 31 & 29 & 29 \\
\hline
\end{tabular}

TABLE 1. Number of iterations required using the optimized Schwarz methods to obtain an error of $10^{-6}$ for a random initial guess and different mesh sizes $h$.

We see that $R_{1}\left(\omega_{1}\right)$ and $R_{2}\left(\omega_{2}\right)$ are in competition with $R_{3}\left(k_{\max }\right)$, and we thus have by the equioscillation principle $\alpha=1-\alpha$ and $\alpha=1 / 2$. Then

$$
R_{1}\left(\omega_{1}, \omega_{1}, \omega_{2}, \varepsilon, C_{1}, C_{2}\right) \simeq 1-\mathcal{O}\left(h^{1 / 2}\right) \simeq R_{3}\left(k_{\max }, \omega_{1}, \omega_{2}, \varepsilon, C_{1}, C_{2}\right)
$$

This is however asymptotically worse than the solution we found for the case $\alpha>\beta$ which was independent of $h$, and hence $\alpha=\beta$ can not lead to the optimal choice asymptotically.

We finally treat the Case $\alpha<\beta$ : here we can use the symmetry of (9); we just have to note that if we consider $\alpha<\beta$ this is equivalent to exchange the constants $c_{1}$ and $c_{2}$ and we have to replace $\varepsilon$ with $\varepsilon^{-1}$. The asymptotic calculation performed for the case $\beta<\alpha$ can then be transformed into this case, which concludes the proof.

\section{NumERICAL EXPERIMENTS}

To illustrate our theoretical results, we perform now numerical experiments on the rectangular domain $\Omega=(-1,1) \times(0,1)$ decomposed into the two sub-domains $\Omega_{1}=(-1,0) \times(0,1)$ and $\Omega_{2}=(0,1) \times(0,1)$. We consider constant coefficients $\varepsilon_{j}, \mu_{j}$ with $j=1,2$. We discretize the Maxwell equation for $\omega=2 \pi$ using a finite volume scheme with mesh size $h=\frac{1}{64}$ based on the classical Yee scheme, see e.g. [23]. We impose on the outer boundaries the impedance condition $\frac{\mathbf{E}}{Z_{j}} \times \mathbf{n}_{j}+\mathbf{n}_{j} \times\left(\mathbf{H} \times \mathbf{n}_{j}\right)=0$, with $j=1,2$. We then use as initial guess each of the Fourier modes $\sin (k \pi y)$ at the interface to see if the discretized algorithm on the bounded domain behaves in a comparable way to our analysis of the convergence factor. We see in Figure 6

a comparison between the theoretical and numerical convergence factors, and also an asymptotic performance study. In the top row, and at the bottom left, the plots show that the numerical convergence factor of the discretized problem on a bounded domain is well predicted by the theoretical convergence factor in Theorem 3 and 5 . On the bottom right, we use a random initial guess and iterate until the error is reduced to $1 e-6$ in the $L^{\infty}$ norm at the interfaces for various mesh sizes $h$. We see that indeed when the mesh is refined and $h$ becomes small, the optimized Schwarz methods corresponding to Theorem 5 have an iteration number that is independent of the mesh size, whereas the optimized Schwarz methods based on Theorem 3 slowly deteriorate when the mesh is refined, at the predicted rate in Theorem 3. These results are also shown in Table 1 , where one can clearly see that Theorem 3 and 5 obtained at the continuous level on the unbounded domain predict well the behavior of the discretized optimized Schwarz method on the bounded domain. We finally show in Table 2 the number of GMRES iterations needed when solving Maxwell's equations with a right hand side equal to one, starting with a zero initial guess and reducing the relative residual to $10^{-6}$. For more numerical experiments, see $[20,22]$.

\section{Conclusion}

We have determined the best choice of parameters in the transmission conditions of optimized Schwarz methods for Maxwell's equations in the presence of discontinuous coefficients, where the discontinuities are aligned with the subdomain interfaces. Using asymptotic analysis, we obtained closed form formulas for these parameters which can easily be used in implementations. Our results showed that with the specific transmission 

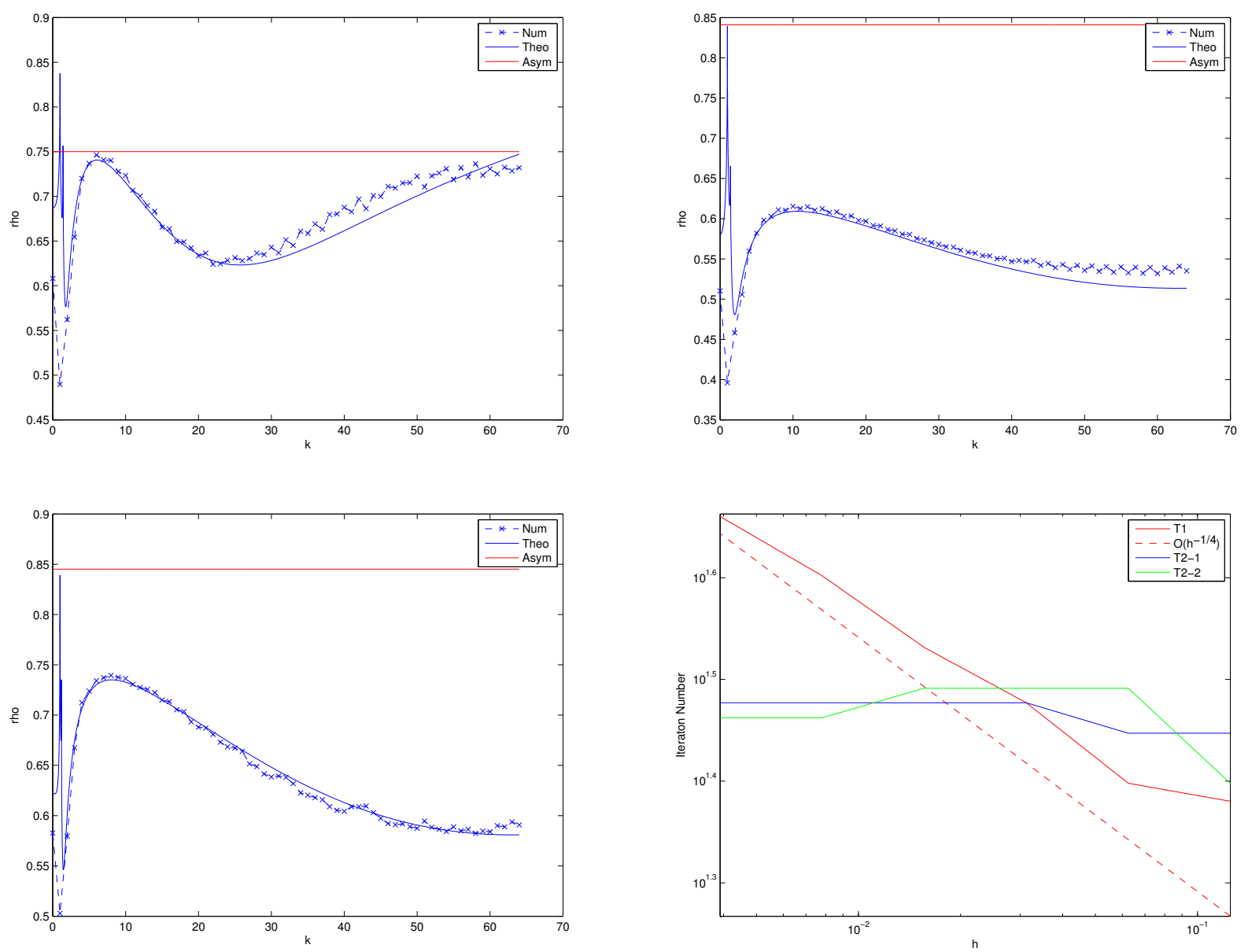

FIgURE 6. Comparison between the theoretical and numerical convergence factors as a function of the Fourier frequency $k$, where we set every Fourier mode $\sin (k \pi y)$ as initial guess and computed the numerical convergence factor for 4 iterations. Top left: case of Theorem 3 with $\varepsilon_{1}=\mu_{1}=\mu_{2}=1, \varepsilon=2$ and $w=\pi$. Top right: first case of Theorem 5 with $\varepsilon_{1}=\mu_{1}=\mu_{2}=1$, $\varepsilon=2$ and $w=\pi$. Bottom left: second case of Theorem 5 with $\varepsilon_{1}=\mu_{1}=\mu_{2}=1, \varepsilon=1.4$ and $w=\pi$. Bottom right: Number of iterations when the mesh size $h$ is refined.

\begin{tabular}{|c|c|c|c|c|c|c|}
\hline$h$ & $\frac{1}{8}$ & $\frac{1}{16}$ & $\frac{1}{32}$ & $\frac{1}{64}$ & $\frac{1}{128}$ & $\frac{1}{256}$ \\
\hline Theorem 3 & 11 & 17 & 21 & 26 & 30 & 35 \\
\hline Theorem 5(Case 1) & 11 & 16 & 19 & 21 & 23 & 24 \\
\hline Theorem 5(Case 2) & 11 & 18 & 23 & 26 & 30 & 34 \\
\hline
\end{tabular}

TABLE 2. Number of iterations required to obtain a relative residual reduction of $10^{-6}$ for different mesh sizes $h$, using the optimized Schwarz method as a preconditionner for GMRES. 
conditions in optimized Schwarz methods which take the physics of the underlying problem into account, one can not just obtain robustness in terms of the jumps in the coefficients, but even benefit from them, obtaining non-overlapping optimized Schwarz methods that converge independent of the mesh parameter $h$, which is not possible if the coefficients do not have jumps.

\section{REFERENCES}

[1] A. Alonso-Rodriguez and L. Gerardo-Giorda. New nonoverlapping domain decomposition methods for the harmonic Maxwell system. SIAM J. Sci. Comput., 28(1):102-122, 2006.

[2] M. El Bouajaji, V. Dolean, M. J. Gander, and S. Lanteri. Comparison of a one and two parameter family of transmission conditions for Maxwell's equations with damping. In Randolph E. Bank, Michael J. Holst, Olof B. Widlund, and Jinchao $\mathrm{Xu}$, editors, Domain Decomposition Methods in Science and Engineering XX, volume 91 of Lecture Notes in Computational Science and Engineering, pages 271-278. Springer, 2013.

[3] Y. Boubendir, X. Antoine, and C. Geuzaine. A quasi-optimal non-overlapping domain decomposition algorithm for the Helmholtz equation. J. Comput. Phys., 231(2):262-280, 2012.

[4] P. Chevalier and F. Nataf. An OO2 (Optimized Order 2) method for the Helmholtz and Maxwell equations. In 10th International Conference on Domain Decomposition Methods in Science and in Engineering, pages 400-407. AMS, 1997.

[5] B. Deprés. Méthodes de décomposition de domains pour les problèms de propagation d'ondes en régime harmonique. PhD thesis, Université Paris IX Dauphine, 1991.

[6] V. Dolean, M. El Bouajaji, M. J. Gander, and S. Lanteri. Optimized Schwarz methods for Maxwell's equations with non-zero electric conductivity. In Domain decomposition methods in science and engineering XIX, volume 78 of Lect. Notes Comput. Sci. Eng., pages 269-276. Springer, Heidelberg, 2011.

[7] V. Dolean, M. El Bouajaji, M. J. Gander, S. Lanteri, and R. Perrussel. Domain decomposition methods for electromagnetic wave propagation problems in heterogeneous media and complex domains. In Domain decomposition methods in science and engineering XIX, volume 78 of Lect. Notes Comput. Sci. Eng., pages 15-26. Springer, Heidelberg, 2011.

[8] V. Dolean, M.J. Gander, and L. Gerardo-Giorda. Optimized Schwarz methods for Maxwell equations. SIAM J. Scient. Comp., 31(3):2193-2213, 2009

[9] V. Dolean, S. Lanteri, and R. Perrussel. A domain decomposition method for solving the three-dimensional time-harmonic Maxwell equations discretized by discontinuous Galerkin methods. J. Comput. Phys., 227(3):2044-2072, 2008.

[10] M. El Bouajaji, V. Dolean, M. J. Gander, and S. Lanteri. Optimized Schwarz methods for the time-harmonic Maxwell equations with damping. SIAM Journal on Scientific Computing, 34(4):A2048-A2071, 2012.

[11] B. Engquist and A. Majda. Absorbing boundary conditions for the numerical simulation of waves. Math. Comp., 31(139):629$651,1977$.

[12] O. G. Ernst and M. J. Gander. Why it is difficult to solve Helmholtz problems with classical iterative methods. In Numerical analysis of multiscale problems, volume 83 of Lect. Notes Comput. Sci. Eng., pages 325-363. Springer, Heidelberg, 2012.

[13] M. J. Gander, L. Halpern, and F. Magoulès. An optimized Schwarz method with two-sided robin transmission conditions for the Helmholtz equation. Int. J. for Num. Meth. in Fluids, 55(2):163-175, 2007.

[14] Martin J. Gander. Optimized Schwarz methods for Helmholtz problems. In Thirteenth international conference on domain decomposition, pages 245-252, 2001.

[15] Martin J. Gander and Hui Zhang. Iterative solvers for the Helmholtz equation: factorizations, sweeping preconditioners, source transfer, single layer potentials, polarized traces, and optimized Schwarz methods, 2016.

[16] M.J. Gander, F. Magoulès, and F. Nataf. Optimized Schwarz methods without overlap for the Helmholtz equation. SIAM J. Sci. Comput., 24(1):38-60, 2002.

[17] David J Griffiths. Introduction to electrodynamics; 4th ed. Pearson, Boston, MA, 2013.

[18] Z. Peng and J. F. Lee. Non-conformal domain decomposition method with second-order transmission conditions for timeharmonic electromagnetics. J. Comput. Physics, 229(16):5615-5629, 2010.

[19] Z. Peng, V. Rawat, and J. F. Lee. One way domain decomposition method with second order transmission conditions for solving electromagnetic wave problems. J. Comput. Physics, 229(4):1181-1197, 2010.

[20] V.Dolean, M. J. Gander, and E. Veneros. Optimized Schwarz methods for Maxwell equations with discontinuous coefficients. In Domain Decomposition Methods in Science and Engineering XXI, Lecture Notes in Computational Science and Engineering. Springer-Verlag, 2013.

[21] V.Dolean, M. J. Gander, and E. Veneros. Schwarz methods for second order Maxwell equations in 3d with coefficient jumps. In Domain Decomposition Methods in Science and Engineering XXII, Lecture Notes in Computational Science and Engineering. Springer-Verlag, 2015.

[22] E. Veneros. Méthodes de décomposition de domaines pour des problèms de propagation d'ondes hétérogènes. PhD thesis, Université de Genève, 2015.

[23] Kane S. Yee. Numerical solution of initial boundary value problems involving Maxwell's equations in isotropic media. IEEE Trans. Antennas and Propagation, pages 302-307, 1966. 